\title{
The epidemiological burden and overall distribution of chronic comorbidities in coronavirus disease-2019 among 202,005 infected patients: evidence from a systematic review and meta-analysis
}

\author{
Rashidul Alam Mahumud ${ }^{1,2}$ (D) . Joseph K. Kamara ${ }^{1,3}$. Andre M. N. Renzaho ${ }^{1,2,4}$
}

Received: 20 April 2020 / Accepted: 7 August 2020 / Published online: 19 August 2020

(c) Springer-Verlag GmbH Germany, part of Springer Nature 2020

\begin{abstract}
Purpose The main purpose of this study was to examine the overall distribution of chronic comorbidities in coronavirus disease-19 (COVID-19) infected populations and the risk of the underlying burden of disease in terms of the case fatality ratio (CFR).

Methods We carried out a systematic review and meta-analysis of studies on COVID-19 patients published before $10^{\text {th }}$ April 2020. Twenty-three studies containing data for 202,005 COVID-19 patients were identified and included in our study. Pooled effects of chronic comorbid conditions and CFR with 95\% confidence intervals were calculated using random-effects models. Results A median age of COVID-19 patients was 56.4 years and 55\% of the patients were male. The most prevalent chronic comorbid conditions were: any type of chronic comorbidity (37\%; 95\% CI 32-41\%), hypertension (22\%; 95\% CI 17-27\%), diabetes (14\%; 95\% CI 12-17\%), respiratory diseases (5\%; 95\% CI 3-6\%), cardiovascular diseases (13\%; 95\% CI 10-16\%) and other chronic diseases (e.g., cancer) (8\%; 95\% CI 6-10\%). Furthermore, 37\% of COVID-19 patients had at least one chronic comorbid condition, $28 \%$ of patients had two conditions, and $19 \%$ of patients had three or more chronic conditions. The overall pooled CFR was 7\% (95\% CI 6-7\%). The crude CFRs increased significantly with increasing number of chronic comorbid conditions, ranging from $6 \%$ for at least one chronic comorbid condition to $13 \%$ for 2 or 3 chronic comorbid conditions, $12 \%$ for 4 chronic comorbid conditions, $14 \%$ for 5 chronic comorbid conditions, and $21 \%$ for 6 or more chronic comorbid conditions. Furthermore, the overall CFRs also significantly increased with higher levels of reported clinical symptoms, ranging from $14 \%$ for at least four symptoms, to $15 \%$ for 5 or 6 symptoms, and $21 \%$ for 7 or more symptoms. Conclusions The chronic comorbid conditions were identified as dominating risk factors, which should be considered in an emergency disease management and treatment choices. There is urgent need to further enhance systematic and real-time sharing of epidemiologic data, clinical results, and experience to inform the global response to COVID-19.
\end{abstract}

Keywords COVID-19 $\cdot$ Coronavirus $\cdot$ Chronic cormobidity $\cdot$ Disease $\cdot$ Fatality

Rashidul Alam Mahumud

r.mahumud@westernsydney.edu.au;

rashidul.icddrb@gmail.com

1 School of Social Sciences, Western Sydney University, Penrith 2751, Australia

2 Translational Health Research Institute, Western Sydney University, Penrith 2751, Australia

3 World Vision International, East Africa Regional Office, Karen, Nairobi, Kenya

4 Maternal, Child and Adolescent Health Program, Burnet Institute, Melbourne 3004, Australia

$\begin{array}{ll}\text { Abbreviations } \\ \text { CFR } & \text { Case fatality ratio } \\ \text { CI } & \text { Confidence intervals } \\ \text { JBI } & \text { Joanna Briggs Institute } \\ \text { WHO } & \text { World Health Organisation }\end{array}$

SARS-CoV-2 Severe acute respiratory syndrome coronavirus 2

\section{Introduction}

On December 31, 2019, China reported a series of pneumonia cases with an unknown cause that was later identified as severe acute respiratory syndrome coronavirus 2 (SARSCoV-2) [1-3]. Subsequently, a novel coronavirus that is 
phylogenetically in the SARS-CoV clade was reported as the causative agent of the outbreak. The disease was named a novel coronavirus disease-2019 (COVID-19) and was declared a global pandemic by the Director General of the World Health Organisation (WHO) on March 11, 2020 [1]. Patients with the disease commonly present with fever, cough, and shortness of breath within an incubation period of 2-14 days [3].

So far, the majority of COVID-19 cases $(80 \%)$ are milder respiratory infections and pneumonia [4]. The risk of severe illness and associated death with COVID-19 infection is considered high among the elderly and individuals with underlying chronic health conditions [4]. Some studies have reported a high proportion of COVID-19 infected individuals (30 to $48 \%$ ) had at least one chronic comorbid condition [2, 5-8], including hypertension [7, 8], cardiovascular diseases [9-11], diabetes [12, 13], respiratory system diseases, and other chronic diseases [14]. Chronic diseases lead to several clinical features with complications, including the proinflammatory state and the reduction of the innate immune response [15, 16]. Chronic comorbid conditions of patients contribute to major clinical challenges in terms of diagnosis, ill health and disease management, which adversely influence treatment choices and outcomes [17]. Ultimately, the severity of comorbidity leads to poor health conditions and outcomes, an increased risk of hospitalisation, and an increased financial burden on the healthcare system [18-20].

Measuring the prevalence of chronic comorbid conditions can be a basis for mitigating complications in patients with COVID-19 infection. Therefore, using a systematic review and meta-analysis, we aimed to estimate the pooled prevalence of comorbidities in all patients and to investigate the risk of underlying diseases in terms of crude case fatality ratio (CFR). Unlike previous systematic review studies [21-23] that focussed on specific regions, our study is unique in that it includes published studies with vast data on COVID-19 patients from different regions. The large pool of data and wide geographical coverage represents various populations giving insight into how different populations and regions respond to COVID-19. We anticipate that our study findings will provide comprehensive understanding of COVID-19 and will inform prevention, control, and response policies and practices.

\section{Methods}

\section{Search strategy}

We conducted a comprehensive search for academic studies that reported in COVID -19 patients published between January 2020 and 9th April 2020 from the following electronic bibliographic databases: PubMed, Scopus, EBSCOhost (CINAHL, Medline), Web of Science, and the first 20 pages of Google Scholar. The following search terms were used with no language restrictions:

["2019 novel coronavirus or COVID-19"] AND ["comorbidities" OR "chronic comorbidity" OR "chronic diseases" OR "diabetes" OR "hypertension" OR "respiratory diseases" OR "cardiovascular diseases" OR "cancer" OR "malignancy" OR "asthma" OR "bronchitis" OR "kidney disease"].

\section{Study participants}

Our study scope comprise of patients that were clinically presented with COVID-19 characteristics and were hospitalised.

\section{Inclusion and exclusion criteria}

Eligible studies were included if they 1) were original articles; 2) published between January 2020 and April 9th, 2020; focused on 3) epidemiological perspective, 4) reported clinical characteristics of the COVID-19 among infected people, and 5) reported the prevalence of chronic comorbid conditions in infected patients. Reviews, editorials, letters, perspectives, commentaries, reports, and studies with 'insufficient related data' were excluded. The reference lists of studies included were checked for eligible studies.

\section{Data extraction}

Data were independently extracted into EndNote libraries by two researchers (RAM and AMNR) who later compared their results. Emerging differences in the data were discussed and resolved by consensus and where the two researchers could not agree, a third researcher (JKK) was consulted for adjudication. Extracted data captured author's name, year, settings, design or approach, age, gender, number of participants, the overall number of deaths, the prevalence of clinical symptoms such as fever, cough, fatigue, polypnea, nausea or vomiting, sputum, dyspnoea, headache, and diarrhea together with chronic comorbid conditions including any type of comorbidity, diabetes, hypertension, respiratory disease and cardiovascular diseases. We also extracted data on CFR.

\section{Study screening and selection}

The eligibility of studies included was determined following a three-stage screening process. The first stage involved screening studies by title to eliminate duplicates. The second stage required reading of abstracts to determine their 
relevance to our study. The third stage necessitated reading of full texts of the retained studies and those that met the set criteria were retained for our study as reflected in Fig. 1. RAM carried out and recorded the above process, and shared the record with AMNR and JKK for verification. Discrepancies were discussed and resolved by consensus.

\section{Quality assessment}

Quality assessment was independently conducted by two authors (RAM and JKK) who applied four quality assessment tools due to the heterogeneity of included studies designs. Discrepancies in the two author's quality assessments were referred to the AMNR for adjudication. The included studies designs were cohort, cross-sectional, case series, and case controls. The four critical appraisal tools applied were from the Joanna Briggs Institute (JBI). The JBI tools have been used widely in academic studies [24-26]. The tools provide a subjective assessment of risk of bias (ranked as low, moderate, or high) [27]. Higher quality indicates greater confidence that future research is unlikely to change or contradict the results, while lower quality indicates higher likelihood that future research may not replicate. The first JBI tool used was the checklist for cohort studies. The checklist assesses critical areas of studies' methodologies for biasness in design, implementation and analysis (Appendix Table 5). The JBI tool for cohort studies was applied to ten cohort studies include in our systematic review and meta-analysis. The second tool of quality assessment used was the JBI checklist for analytical cross-sectional studies. The checklist was applied to two cross-sectional studies included in this review. The checklist consists of eight items that enables the critical appraisal of studies for potential biasness (Appendix Table 6). The third JBI tool applied was the checklist for case series and this was applied to ten studies included in this review (Appendix Table 7). The checklist comprises of ten items that examines the criteria for inclusion in the case series, reliability of the condition of measured, validity of methods used in identification of the condition for participants, consecutive inclusion of participants, completeness of participants inclusion, clarity of reporting on study demographics, clarity of clinical information reporting, clarity of results reporting and the clarity of information on the study site(s). The fourth and last tool used was the JBI checklist for case control studies and was applied to one study included in our review (Appendix Table 8). The checklist comprises of ten items measuring comparability of study groups, matching of cases and controls, identification criteria for cases and controls, reliability and validity of exposure measurement, similarities in exposure measurement for case and control, identification of confounding factors, addressing the confounding factors, assessment of outcomes, length of exposure period and statistical analysis.

\section{Data analysis}

The case fatality rate (CFR) was derived for COVID-19 infected populations for all studies, which describes the ratio of deaths to cases. We investigated the association between
Fig. 1 Steps of study selection procedures

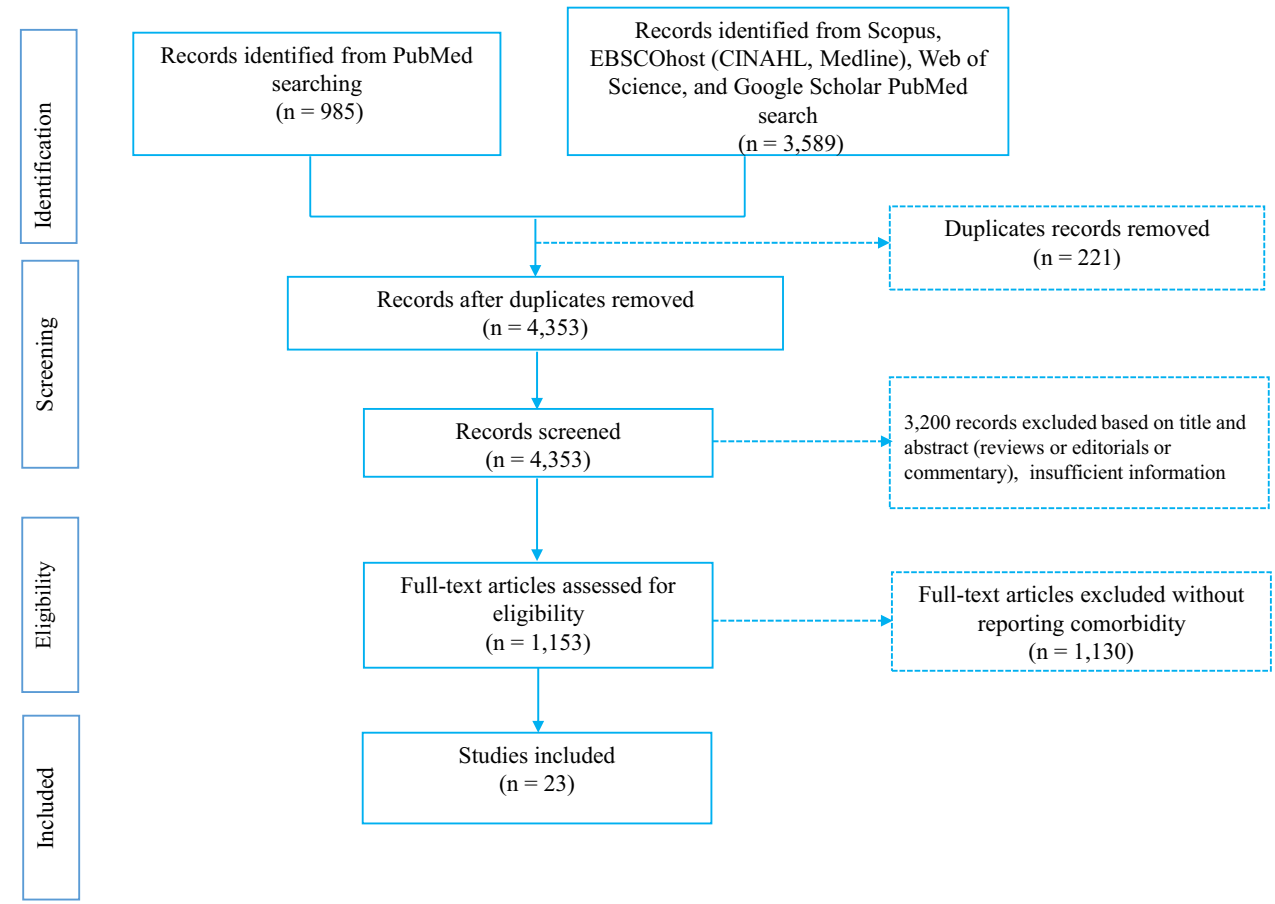


the case fatality ratio and the chronic comorbid conditions among the COVID-19 infected population. Given the high heterogeneity between studies $\left(I^{2}>50 \%\right)$ [28], we used random effect models with the $95 \%$ confidence intervals (CI) of estimates, complemented with a sensitivity analysis to examine the effects of outliers. The $I^{2}$ statistic enabled us determine whether the percentage of variance was attributable to the heterogeneity of the data in studies included using the random-effects model. We used forest plots to show the distribution of chronic comorbid conditions in coronavirus disease-2019 patients. All statistical analyses were performed by STATA/SE version 14.0. Furthermore, publication bias was assessed using the Begg's test [29] and Egger's test [30]. Both of the tests are widely used to assess the tendency for the effects estimated in small sample size studies to differ from those estimated in larger studies. The risk of publication bias was analysed in terms of chronic comorbid conditions and CFR due to number of comorbidities. Subgroup and meta-regression analyses were conducted to verify the association of CFR and chronic comorbid conditions among COVID-19 infected patient, where moderate or higher heterogeneity was reported [31,32]. Additionally, a permutation test was employed based on Monte Carlo simulation by controlling the risk of spurious findings from meta-regression [33], wherein unadjusted and adjusted estimation approaches were also used to calculate $p$ values in meta-regression. In a permutation test, the covariates were randomly reallocated to the outcomes for 10,000 times to adjust for multiple testing to compare the observed t-statistic for every covariate with the largest $t$-statistic for any covariate in each random permutation.

\section{Results}

\section{Description of studies included}

Our primary search of databases yielded 4453 studies of which 23 met our criteria and were included in this study (Fig. 1), giving a total sample of 202,005 coronavirus disease-2019 confirmed cases and 3,895 confirmed deaths (Table 1). The average age of participants $(55 \%$ male and $45 \%$ female) was 56.4 years, whereas the overall incubation period was 7.8 days.

\section{Distribution of comorbidities}

Our findings suggest the predominant clinical symptoms were fever (87.5\%; 95\% CI 87.5-87.5\%), cough (57.1\%; 95\% CI 57.1-57.1\%), and fatigue $(32.7 \%$; $95 \%$ CI $32.7-32.7 \%$ ) (Table 2). Our analysis shows, the most prevalent of chronic comorbid conditions were (Fig. 2): any type of chronic comorbidity (37\%; 95\% CI 32-41\%; $p<0.001)$, hypertension (22\%; 95\% CI 17-27\%; $p<0.001)$, diabetes (14\%; 95\% CI 12-17\%; $p<0.001)$, cardiovascular $(13 \%$; 95\% CI: $10-16 \% ; p<0.001)$, respiratory disease $(5 \% ; 95 \%$ CI $3-6 \% ; p<0.001)$, and other chronic diseases $(8 \%$; $95 \%$ CI $6-10 \% ; p<0.001)$.

\section{Mortality among COVID-19 patients}

The overall pooled CFR was 7\% (95\% CI 6-7\%; $p<0.001$; $I^{2}=97.7 \%$ ) (3895 deaths among 202,005 confirmed cases) and significantly increased $(p=0.01)$ with higher levels of chronic comorbid conditions (Fig. 3), ranging from $7 \%$ for at least one chronic comorbid condition $(p<0.001)$, to $13 \%$ for 2 or 3 chronic comorbid conditions $(p<0.001), 12 \%$ for 4 chronic comorbid conditions $(p<0.001), 14 \%$ for 5 chronic comorbid conditions $\left(p<0.001, I^{2}=98.2 \%\right)$, and $21 \%$ for 6 or more chronic comorbid conditions $(p<0.001)$. Furthermore, the overall CFRs also significantly increased with higher levels of reported clinical symptoms (Fig. 4), ranging from $14 \%$ for at least four symptoms $(p<0.001)$, to $15 \%$ for 5 or 6 symptoms ( $p<0.001$ ), and $21 \%$ for 7 or more symptoms $(p<0.001)$.The results of Egger's test were presented in terms of bias coefficient (Table 3). Publication bias was only observed in studies identified to estimate the prevalence of any type of chronic disease $(p=0.002)$, hypertension $(p<0.001)$, diabetes $(p=0.015)$ and CVD $(p=0.050)$. However, the $p$ values for the Egger's test were 0.177 (for respiratory system disease) and 0.120 (for other chronic disease), respectively, denoting absent of publication bias (Table 3).

Our analysis shows that a high heterogeneity $\left(I^{2}>75 \%\right)$ was also observed for meta-regression $(96.86 \%)$ in terms of CFR (Table 3). To examine the sources of heterogeneity, we conducted stratified analysis across chronic comorbid conditions, study design (e.g., case series vs cohort vs crosssectional), sample size (i.e., $\leq 200$ or $>200$ ), age of the total sample (i.e., $<50$ years or $\geq 50$ years). We found that the risk of mortality varied and significantly associated with chronic comorbid conditions, aged patients and increased incubation period. For instance, the CFR was significantly higher for COVID-19 patients with pre-existing any type of chronic disease (beta, $\beta=0.014, p=0.007$ ), hypertension $(\beta=0.055, p=0.054)$, diabetes $(\beta=0.188, p=0.023)$, respiratory system diseases $(\beta=0.331, p=0.022)$. A similar association was also observed among patients with higher levels of chronic comorbid conditions $(p<0.050)$ and increased incubation period in day $(p=0.043)$ (Table 4).

\section{Quality of included studies}

Our findings suggest that eleven $(n=11)$ of the included studies were of high quality implying that they were robust studies; twelve of the studies were ranked as medium 
Table 1 Characteristics of selected studies

\begin{tabular}{|c|c|c|c|c|c|c|c|c|}
\hline $\begin{array}{l}\text { Selected studies by } \\
\text { study design }\end{array}$ & Settings & $\begin{array}{l}\text { Infected } \\
\text { population } \\
(n)\end{array}$ & Age (year) & $\%$ of male & $\begin{array}{l}\text { Incuba- } \\
\text { tion period } \\
\text { (days) }\end{array}$ & $\begin{array}{l}\text { Total } \\
\text { number of } \\
\text { deaths, } n\end{array}$ & $\begin{array}{l}\text { Case fatality ratio } \\
(95 \% \mathrm{CI})\end{array}$ & $\%$ Weight \\
\hline \multicolumn{9}{|l|}{ Cohort study } \\
\hline Zhou et al. [34] & Wuhan, China & 191 & 56.00 & 62.00 & 11.00 & 54 & $0.28(0.22,0.35)$ & 1.33 \\
\hline Zhang et al. [12] & Wuhan, Hubei & 258 & 64.00 & 53.50 & 12.00 & 15 & $0.06(0.03,0.09)$ & 4.50 \\
\hline Wu et al. [35] & Wuhan, China & 188 & 51.90 & 63.60 & 9.00 & 43 & $0.23(0.17,0.30)$ & 1.49 \\
\hline Cheng et al. [36] & Wuhan, China & 701 & 63.00 & 52.40 & 10.00 & 113 & $0.16(0.13,0.19)$ & 4.76 \\
\hline Chen et al. [6] & Huanan, China & 99 & 55.50 & 68.00 & 6.50 & 11 & $0.11(0.06,0.19)$ & 1.41 \\
\hline Fu et al. [37] & Wuhan, Hubei & 200 & 65.00 & 49.30 & 8.00 & 34 & $0.17(0.12,0.23)$ & 1.89 \\
\hline Shi et al. [38] & China & 416 & 64.10 & 49.30 & 5.00 & 57 & $0.14(0.11,0.17)$ & 3.75 \\
\hline Guan et al. [39] & China & 1099 & 47.00 & 58.10 & 4.00 & 15 & $0.01(0.01,0.02)$ & 10.21 \\
\hline Yan et al. [40] & Wuhan, China & 193 & 64.00 & 59.10 & 13.00 & 108 & $0.56(0.49,0.63)$ & 1.13 \\
\hline Wang et al. [41] & Wuhan, China & 69 & 42.00 & 46.00 & 4.00 & 5 & $0.07(0.02,0.16)$ & 1.44 \\
\hline Sub-total (pooled) & $n=10$ & 3414 & 57.24 & 56.13 & 8.25 & 455 & $0.18(0.10,0.25)$ & 31.90 \\
\hline \multicolumn{9}{|l|}{ Cross-sectional study } \\
\hline Pan et al. [42] & China & 204 & 52.90 & 52.40 & 8.10 & 36 & $0.18(0.13,0.24)$ & 1.88 \\
\hline Cao et al. [43] & Shanghai, China & 198 & 50.10 & 51.00 & 4.00 & 1 & $0.01(0.00,0.03)$ & 9.42 \\
\hline Sub-total (pooled) & $n=2$ & 402 & 51.50 & 51.70 & 6.05 & 37 & $0.01(0.00,0.02)$ & 11.30 \\
\hline \multicolumn{9}{|l|}{ Case series study } \\
\hline Guan et al. [44] & China & 1590 & 48.90 & 57.30 & 3.60 & 50 & $0.03(0.02,0.04)$ & 9.78 \\
\hline Wang et al. [5] & Wuhan, China & 138 & 56.00 & 54.30 & 5.00 & 6 & $0.04(0.02,0.09)$ & 3.60 \\
\hline Chen et al. [45] & Wuhan, China & 799 & 62.00 & 62.00 & 10.00 & 113 & $0.14(0.12,0.17)$ & 5.41 \\
\hline Liu et al. [46] & Hubei, China & 137 & 57.00 & 44.50 & 7.00 & 16 & $0.12(0.07,0.18)$ & 1.79 \\
\hline CDC [8] & USA & 122,653 & - & - & - & 2112 & $0.02(0.02,0.02)$ & 11.05 \\
\hline McMichael et al. [2] & Washington, USA & 167 & 72.00 & 32.90 & 9.00 & 35 & $0.21(0.15,0.28)$ & 1.42 \\
\hline Wu et al. [47] & China & 72,314 & 44.00 & - & - & 1023 & $0.01(0.01,0.02)$ & 11.05 \\
\hline Guo et al. [48] & Wuhan, China & 174 & 59.00 & 43.70 & 7.00 & 9 & $0.05(0.02,0.10)$ & 3.77 \\
\hline Wan et al. [49] & Chongqing, China & 135 & 47.00 & 53.30 & 5.00 & 1 & $0.01(0.00,0.04)$ & 8.05 \\
\hline Huang et al. [7] & Wuhan, China & 41 & 49.00 & 73.00 & 8.00 & 6 & $0.15(0.06,0.29)$ & 0.50 \\
\hline Sub-total (pooled) & $n=10$ & 198,148 & 54.99 & 52.63 & 6.83 & 3371 & $0.03(0.02,0.04)$ & 56.43 \\
\hline \multicolumn{9}{|l|}{ Case-control study } \\
\hline Su et al. [50] & Huanan, China & 41 & 70.00 & 75.00 & 14.00 & 32 & $0.78(0.62,0.89)$ & 0.37 \\
\hline Total & $N=23$ & 202,005 & 56.38 & 55.27 & 7.77 & 3895 & $0.07(0.06,0.07)$ & 100.00 \\
\hline $\begin{array}{l}\text { I-squared }\left(I^{2}\right), \%(p \\
\text { value) }\end{array}$ & & & & & & & $97.69 \%(p<0.001)$ & \\
\hline
\end{tabular}

quality implying that their methods were of moderate quality based on the JBI quality assessment criteria. There was no study excluded based on poor scoring on the quality assessment scales. Notably, one $(n=1)$ cohort study [12] was assed as high on the JBI checklist for cohort studies; the rest $(n=9)$ studies were found to be of medium quality on the same scale [6,34-41]. Neither of the ten $(n=10)$ cohort studies articulated how they dealt with confounding factors nor did they articulate strategies to address incomplete follow-up of participants. Furthermore, only two $(n=2)$ of the cohort studies reported that their followup time was sufficient and long enough to observe outcomes. Furthermore, the two cross-sectional studies [42,
43 ] included in our study scored seven out of eight $(n=8)$ items on the JBI quality assessment checklist used. Both studies did not state how they dealt with confounding factors. Eight out of ten $(n=8 / 10)$ case series studies included $[2,5,7,8,44-49]$, ware found to be of high quality based on the JBI quality assessment scale for case series. However, four $(n=4)$ of the case series studies [2, 8, 47, 48] statistical analyses were assessed to be unsatisfactory. Lastly, the only one case-control study [50] included in our review was found to be of medium quality with eight $(n=8 / 10)$ items on the JBI quality assessment scale of case controls. 
Table 2 Distribution of reported symptoms in coronavirus disease-2019 infected populations

\begin{tabular}{|c|c|c|c|c|c|c|c|c|c|}
\hline \multirow{3}{*}{$\begin{array}{l}\text { Selected } \\
\text { studies }\end{array}$} & \multicolumn{9}{|c|}{ Most reported symptoms in the studies } \\
\hline & Fever & Cough & Fatigue & Polypnea & $\begin{array}{l}\text { Nausea or } \\
\text { vomiting }\end{array}$ & Sputum & Dyspnoea & Headache & Diarrhea \\
\hline & $\%(95 \% \mathrm{CI})$ & $\%(95 \% \mathrm{CI})$ & $\%(95 \% \mathrm{CI})$ & $\%(95 \% \mathrm{CI})$ & $\%(95 \% \mathrm{CI})$ & $\%(95 \% \mathrm{CI})$ & $\%(95 \% \mathrm{CI})$ & $\%(95 \% \mathrm{CI})$ & $\%(95 \% \mathrm{CI})$ \\
\hline \multicolumn{10}{|l|}{ Cohort study } \\
\hline $\begin{array}{l}\text { Zhou et al. } \\
\text { [34] }\end{array}$ & $\begin{array}{l}94.00(93.45 \\
94.55)\end{array}$ & $\begin{array}{c}79.00(78.44 \\
79.55)\end{array}$ & $\begin{array}{c}23.00(22.44 \\
23.55)\end{array}$ & - & $\begin{array}{l}4.00(3.44, \\
4.55)\end{array}$ & $\begin{array}{l}23.00(22.44 \\
23.55)\end{array}$ & - & - & $\begin{array}{l}5.00(4.45, \\
5.55)\end{array}$ \\
\hline $\begin{array}{c}\text { Zhang et al. } \\
\text { [12] }\end{array}$ & $\begin{array}{l}82.20(82.09 \\
82.31)\end{array}$ & $\begin{array}{c}67.10(66.98 \\
67.21)\end{array}$ & $\begin{array}{l}38.00(37.88, \\
38.11)\end{array}$ & $\begin{array}{c}48.10(47.98 \\
48.21)\end{array}$ & $\begin{array}{l}8.50(8.38 \\
8.61)\end{array}$ & $\begin{array}{l}5.40(5.28 \\
5.51)\end{array}$ & $\begin{array}{c}48.10(47.98 \\
48.21)\end{array}$ & $\begin{array}{c}10.90(10.78 \\
11.01)\end{array}$ & $\begin{array}{l}21.30(21.18, \\
21.41)\end{array}$ \\
\hline $\begin{array}{l}\text { Wu et al. } \\
\text { [35] }\end{array}$ & $\begin{array}{l}92.60(92.15 \\
93.05)\end{array}$ & $\begin{array}{c}83.50(83.05 \\
83.94)\end{array}$ & $\begin{array}{l}32.50(32.05, \\
32.95)\end{array}$ & $\begin{array}{c}38.30(37.85 \\
38.74)\end{array}$ & - & $\begin{array}{c}39.40(39.95 \\
39.84)\end{array}$ & $\begin{array}{l}12.80(12.35 \\
13.25)\end{array}$ & $\begin{array}{l}33.50(33.05 \\
33.94)\end{array}$ & - \\
\hline $\begin{array}{l}\text { Cheng et al. } \\
\text { [36] }\end{array}$ & $\begin{array}{l}32.50(32.18 \\
32.82)\end{array}$ & - & - & - & - & - & - & - & - \\
\hline $\begin{array}{l}\text { Chen et al. } \\
\text { [6] }\end{array}$ & $\begin{array}{c}83.00(82.78, \\
83.22)\end{array}$ & $\begin{array}{c}82.00(81.78 \\
82.21)\end{array}$ & - & - & $\begin{array}{l}1.00(0.78 \\
1.22)\end{array}$ & - & - & $\begin{array}{l}8.00(7.78, \\
8.21)\end{array}$ & $\begin{array}{l}2.00(1.78, \\
2.22)\end{array}$ \\
\hline $\begin{array}{c}\text { Fu et al. } \\
\text { [37] }\end{array}$ & $\begin{array}{l}88.00(87.67, \\
88.33)\end{array}$ & $\begin{array}{c}46.30(45.96 \\
46.63)\end{array}$ & $\begin{array}{l}52.20(51.86, \\
52.53)\end{array}$ & - & - & - & - & - & $\begin{array}{l}59.70(59.36, \\
60.03)\end{array}$ \\
\hline $\begin{array}{c}\text { Shi et al. } \\
\text { [38] }\end{array}$ & $\begin{array}{l}80.30(80.03 \\
80.57)\end{array}$ & $\begin{array}{c}34.60(34.33 \\
34.86)\end{array}$ & $\begin{array}{c}13.20(12.93, \\
13.46)\end{array}$ & - & - & - & - & $\begin{array}{l}2.20(1.93 \\
2.47)\end{array}$ & $\begin{array}{l}3.80(3.53, \\
4.07)\end{array}$ \\
\hline $\begin{array}{l}\text { Guan et al. } \\
\text { [39] }\end{array}$ & - & $\begin{array}{c}67.80(67.77 \\
67.82)\end{array}$ & $\begin{array}{c}38.10(38.07, \\
38.12)\end{array}$ & $\begin{array}{c}18.70(18.67 \\
18.72)\end{array}$ & $\begin{array}{l}4.80(4.77 \\
4.83)\end{array}$ & & - & $\begin{array}{c}13.60(13.57 \\
13.63)\end{array}$ & $\begin{array}{l}3.80(3.77 \\
3.83)\end{array}$ \\
\hline $\begin{array}{l}\text { Yan et al. } \\
\text { [40] }\end{array}$ & $\begin{array}{l}89.60(88.50 \\
90.69)\end{array}$ & $\begin{array}{c}69.90(68.80 \\
71.99)\end{array}$ & $\begin{array}{l}52.30(51.20, \\
53.39)\end{array}$ & $\begin{array}{l}5.00(3.90, \\
6.09)\end{array}$ & - & - & $\begin{array}{l}59.60(58.50 \\
60.69)\end{array}$ & $\begin{array}{c}10.90(9.80, \\
11.99)\end{array}$ & $\begin{array}{l}26.40(25.30, \\
27.49)\end{array}$ \\
\hline $\begin{array}{l}\text { Wang et al. } \\
\text { [41] }\end{array}$ & $\begin{array}{c}87.00(86.86, \\
87.14)\end{array}$ & $\begin{array}{l}55.00(54.85 \\
55.14)\end{array}$ & $\begin{array}{l}42.00(41.85, \\
42.14)\end{array}$ & $\begin{array}{l}9.00(8.85, \\
9.14)\end{array}$ & - & - & $\begin{array}{c}29.00(28.85 \\
29.14)\end{array}$ & $\begin{array}{c}14.00(13.85 \\
14.14)\end{array}$ & $\begin{array}{c}14.00(13.86, \\
14.14)\end{array}$ \\
\hline $\begin{array}{l}\text { Sub-total } \\
\text { (pooled) }\end{array}$ & $\begin{array}{c}81.56(81.49 \\
81.64)\end{array}$ & & $\begin{array}{c}38.03(38.01 \\
38.06)\end{array}$ & $\begin{array}{c}19.92(19.89 \\
19.95)\end{array}$ & $\begin{array}{c}4.93(4.91, \\
4.96)\end{array}$ & $\begin{array}{l}8.06(7.94, \\
8.17)\end{array}$ & $\begin{array}{c}39.68(39.60 \\
39.78)\end{array}$ & $\begin{array}{c}13.36(13.34 \\
13.39)\end{array}$ & $\begin{array}{l}5.29(5.26 \\
5.32)\end{array}$ \\
\hline \multicolumn{10}{|c|}{ Cross-sectional study } \\
\hline $\begin{array}{l}\text { Pan et al. } \\
\text { [42] }\end{array}$ & $\begin{array}{l}92.23(91.88 \\
92.57)\end{array}$ & $\begin{array}{c}34.00(33.65 \\
34.35)\end{array}$ & - & - & - & - & - & $\begin{array}{l}14.56(14.21 \\
14.91)\end{array}$ & $\begin{array}{c}33.98(33.63, \\
34.32)\end{array}$ \\
\hline $\begin{array}{c}\text { Cao et al. } \\
\text { [43] }\end{array}$ & $\begin{array}{c}86.90(86.89 \\
86.91)\end{array}$ & $\begin{array}{c}46.40(46.39 \\
46.41)\end{array}$ & $\begin{array}{c}31.30(31.29, \\
31.30)\end{array}$ & - & - & $\begin{array}{l}23.20(23.19 \\
23.21)\end{array}$ & - & $\begin{array}{l}12.10(12.09 \\
12.11)\end{array}$ & $\begin{array}{l}4.40(4.39, \\
4.41)\end{array}$ \\
\hline $\begin{array}{l}\text { Sub-total } \\
\quad \text { (pooled) }\end{array}$ & & & $\begin{array}{l}31.30(31.29 \\
31.31)\end{array}$ & - & & $\begin{array}{l}23.20(23.19 \\
23.21)\end{array}$ & & $\begin{array}{l}12.10(12.09 \\
12.11)\end{array}$ & $\begin{array}{l}4.42(4.41, \\
4.43)\end{array}$ \\
\hline \multicolumn{10}{|c|}{ Case-series study } \\
\hline $\begin{array}{l}\text { Guan et al. } \\
\text { [44] }\end{array}$ & $\begin{array}{l}88.00(87.94 \\
88.06)\end{array}$ & $\begin{array}{c}70.20(70.13 \\
70.26)\end{array}$ & $\begin{array}{c}42.80(42.73, \\
42.86)\end{array}$ & $\begin{array}{c}14.70(14.63 \\
14.76)\end{array}$ & $\begin{array}{l}5.80(5.73, \\
5.86)\end{array}$ & - & - & $\begin{array}{c}15.40(15.34 \\
15.46)\end{array}$ & $\begin{array}{l}4.20(4.13, \\
4.26)\end{array}$ \\
\hline $\begin{array}{l}\text { Wang et al. } \\
\text { [5] }\end{array}$ & $\begin{array}{l}98.60(98.52 \\
98.68)\end{array}$ & $\begin{array}{l}59.40(59.31 \\
59.48)\end{array}$ & $\begin{array}{c}69.60(69.51 \\
69.68)\end{array}$ & $\begin{array}{c}17.40(17.31 \\
17.48)\end{array}$ & $\begin{array}{l}3.60(3.51, \\
3.68)\end{array}$ & $\begin{array}{l}2.20(2.11, \\
2.29)\end{array}$ & $\begin{array}{l}31.20(31.11 \\
31.29)\end{array}$ & $\begin{array}{l}6.50(6.42 \\
6.59)\end{array}$ & $\begin{array}{c}10.10(10.01, \\
10.19)\end{array}$ \\
\hline $\begin{array}{l}\text { Chen et al. } \\
\text { [45] }\end{array}$ & $\begin{array}{l}91.00(90.72 \\
91.28)\end{array}$ & $\begin{array}{l}68.00(67.72 \\
68.28)\end{array}$ & $\begin{array}{l}50.00(49.72, \\
50.27)\end{array}$ & $\begin{array}{l}4.00(3.72 \\
4.27)\end{array}$ & $\begin{array}{l}9.00(8.72 \\
9.28)\end{array}$ & $\begin{array}{l}30.00(29.72 \\
30.28)\end{array}$ & $\begin{array}{c}44.00(43.72 \\
44.29)\end{array}$ & $\begin{array}{l}11.00(10.72 \\
11.28)\end{array}$ & $\begin{array}{c}28.00(27.72, \\
28.28)\end{array}$ \\
\hline $\begin{array}{l}\text { McMichae } \\
\text { et al. [2] }\end{array}$ & - & - & - & - & - & - & - & - & - \\
\hline $\begin{array}{l}\text { Wu et al. } \\
\text { [47] }\end{array}$ & - & - & - & - & - & - & - & - & - \\
\hline $\begin{array}{l}\text { Guo et al. } \\
\text { [48] }\end{array}$ & $\begin{array}{c}78.20(78.09 \\
78.30)\end{array}$ & $\begin{array}{c}32.20(32.09 \\
32.30)\end{array}$ & $\begin{array}{c}27.00(26.89 \\
27.10)\end{array}$ & $\begin{array}{l}5.20(5.09 \\
5.30)\end{array}$ & - & - & - & $\begin{array}{l}6.90(6.79, \\
7.00)\end{array}$ & $\begin{array}{l}12.10(11.99, \\
12.20)\end{array}$ \\
\hline $\begin{array}{l}\text { Wan et al. } \\
\text { [49] }\end{array}$ & $\begin{array}{l}88.90(88.89, \\
88.92)\end{array}$ & $\begin{array}{c}76.50(76.48 \\
76.51)\end{array}$ & $\begin{array}{l}32.50(32.48, \\
32.51)\end{array}$ & $\begin{array}{c}17.70(17.68 \\
17.71)\end{array}$ & - & $\begin{array}{l}8.80(8.78, \\
8.82)\end{array}$ & $\begin{array}{c}13.30(13.28 \\
13.31)\end{array}$ & $\begin{array}{l}32.50(32.48 \\
32.52)\end{array}$ & $\begin{array}{c}13.30(13.28, \\
13.32)\end{array}$ \\
\hline $\begin{array}{l}\text { Liu et al. } \\
\text { [46] }\end{array}$ & $\begin{array}{l}48.20(47.97 \\
48.43)\end{array}$ & $\begin{array}{c}32.10(31.87 \\
32.32)\end{array}$ & $\begin{array}{l}32.10(31.87, \\
32.32)\end{array}$ & - & - & - & $\begin{array}{c}19.00(18.77 \\
19.22)\end{array}$ & $\begin{array}{l}9.50(9.27 \\
9.73)\end{array}$ & $\begin{array}{l}8.00(7.77 \\
8.23)\end{array}$ \\
\hline CDC [8] & - & - & - & - & - & - & - & - & - \\
\hline $\begin{array}{l}\text { Huang et al. } \\
\text { [7] }\end{array}$ & $\begin{array}{l}98.00(97.71 \\
98.29)\end{array}$ & $\begin{array}{c}76.00(75.71 \\
76.28)\end{array}$ & $\begin{array}{l}44.00(43.71, \\
44.28)\end{array}$ & - & - & $\begin{array}{l}28.00(27.71 \\
28.29)\end{array}$ & $\begin{array}{l}55.00(54.71 \\
55.29)\end{array}$ & $\begin{array}{l}8.00(7.71, \\
8.29)\end{array}$ & $\begin{array}{l}3.00(2.71, \\
3.29)\end{array}$ \\
\hline $\begin{array}{l}\text { Sub-total } \\
\text { (pooled) }\end{array}$ & $\begin{array}{c}88.79(88.77 \\
88.80)\end{array}$ & $\begin{array}{c}74.74(74.72 \\
74.76)\end{array}$ & $\begin{array}{c}33.94(33.93, \\
33.96)\end{array}$ & $\begin{array}{c}17.27(17.26 \\
17.29)\end{array}$ & & $\begin{array}{c}8.71(8.70 \\
8.73)\end{array}$ & $\begin{array}{c}14.01(13.99 \\
14.02)\end{array}$ & $\begin{array}{c}30.31(30.29 \\
30.32)\end{array}$ & $\begin{array}{c}12.73(12.72, \\
12.75)\end{array}$ \\
\hline
\end{tabular}


Table 2 (continued)

\begin{tabular}{|c|c|c|c|c|c|c|c|c|c|}
\hline \multirow{3}{*}{$\begin{array}{l}\text { Selected } \\
\text { studies }\end{array}$} & \multicolumn{9}{|c|}{ Most reported symptoms in the studies } \\
\hline & Fever & Cough & Fatigue & Polypnea & $\begin{array}{l}\text { Nausea or } \\
\text { vomiting }\end{array}$ & Sputum & Dyspnoea & Headache & Diarrhea \\
\hline & $\%(95 \% \mathrm{CI})$ & $\%(95 \% \mathrm{CI})$ & $\%(95 \% \mathrm{CI})$ & $\%(95 \% \mathrm{CI})$ & $\%(95 \% \mathrm{CI})$ & $\%(95 \% \mathrm{CI})$ & $\%(95 \% \mathrm{CI})$ & $\%(95 \% \mathrm{CI})$ & $\%(95 \% \mathrm{CI})$ \\
\hline \multicolumn{10}{|c|}{ Case-control study } \\
\hline $\begin{array}{l}\text { Su et al. } \\
\text { [50] }\end{array}$ & $\begin{array}{c}81.30(79.77 \\
82.83)\end{array}$ & $\begin{array}{c}59.30(57.77 \\
60.83)\end{array}$ & $\begin{array}{l}43.80(42.27, \\
45.33)\end{array}$ & - & $\begin{array}{l}3.10(1.57, \\
4.63)\end{array}$ & - & - & $\begin{array}{l}15.00(13.47 \\
16.53)\end{array}$ & $\begin{array}{c}3.10(1.57, \\
4.63)\end{array}$ \\
\hline $\begin{array}{l}\text { Inverse vari- } \\
\text { ance }(\mathrm{I}-\mathrm{V}) \\
\text { pooled esti- } \\
\text { mate, } \% \\
(95 \% \mathrm{CI})\end{array}$ & $\begin{array}{c}87.47(87.46 \\
87.48)\end{array}$ & $\begin{array}{c}57.09(57.08, \\
57.09)\end{array}$ & $\begin{array}{l}32.73(32.72, \\
32.74)\end{array}$ & $\begin{array}{c}17.87(17.86 \\
17.89)\end{array}$ & $\begin{array}{l}4.98(4.96 \\
5.01)\end{array}$ & $\begin{array}{c}18.44(18.43 \\
18.45)\end{array}$ & $\begin{array}{c}14.67(14.66 \\
14.69)\end{array}$ & $\begin{array}{c}17.86(17.85 \\
17.87)\end{array}$ & $\begin{array}{c}7.08(7.07 \\
7.09)\end{array}$ \\
\hline $\begin{array}{l}I \text {-squared } \\
I^{2}, \%\end{array}$ & $100 \%$ & $99 \cdot 6 \%$ & $100 \%$ & $99.9 \%$ & $99.9 \%$ & $97 \cdot 9 \%$ & $100 \%$ & $100 \%$ & $100 \%$ \\
\hline ( $p$ value) & $(p<0 \cdot 001)$ & $(p<0.001)$ & $(p<0.001)$ & $(p<0.001)$ & $(p<0.001)$ & $(p<0.001)$ & $(p<0.001)$ & $(p<0.001)$ & $(p<0.001)$ \\
\hline
\end{tabular}

$p$ value for heterogeneity, $C I$ confidence interval

\section{Discussion}

Our meta-analysis was based on data from 23 studies with laboratory-confirmed 202,005 COVID-19 infected patients. The patients average age was 56.4 years and almost $55 \%$ of the patients were men. This finding suggests age and gender were critical determinants in COVID-19 infection. This finding is consistent with earlier studies that found approximately 60 to $70 \%$ of COVID-19 patients were elderly men [51-53].

Our findings suggest that the most predominant clinical symptoms of COVID-19 were fever (87.5\%), cough $(57.1 \%)$, and fatigue $(32.7 \%)$. This finding differs from earlier studies that associated COVID-19 symptoms with viral pneumonia, fatigue, and lymphopenia $[3,14,16,51$, 53, 54]. A recent study observed that $10 \%$ of COVID-19 patients presented with diarrhea and nausea initially [5]. An earlier study conducted in China argued that nausea or vomiting and diarrhea were low (5.0\% and 3.8\%) [44]. However, another study reported diarrhea $(80 \%)$ and nausea $(50 \%)$ were more common symptoms in COVID-19 infected populations [51]. Such discrepancies could be a result of limited understanding of the disease, methodological inconsistencies, inadequate classification of the disease, inadequate differential diagnosis and, classification biases caused by small sample sizes. In addition, the discrepancies in clinical symptoms could lead to misdiagnosis of the symptoms when patients seek clinical attention. A misdiagnosis of the symptoms implies patients who should be placed in high risk isolation wards could be mixed with other patients causing further transmissions to non COVID-19 patients and their careers.

Our analysis shows, the most prevalent of chronic comorbid conditions among COVID-19 infected population were at least one underlying chronic comorbid condition (37\%), hypertension (22\%), diabetes (14\%), cardiovascular diseases (13\%), respiratory disease (5\%), and other chronic diseases (8\%). This is consistent with a previous studies that noted $40 \%$ of patients had at least one underlying chronic disease [55] and that approximately $23 \%$ of infected individuals suffered from hypertension [11], followed by diabetes mellitus (17\%), and cardiovascular diseases (10\%) [55]. Chronic diseases lead to several clinical features with severe complications, including the proinflammatory state, and the reduction of the innate immune response [15, 16]. For instance, diabetes mellitus occurs in part due to accumulation of stimulated innate immune cells in metabolic tissues that contribute to the release of inflammatory mediators, particularly, IL- $1 \beta$ and TNF $\alpha$, which develop systemic insulin resistance and $\beta$-cell damage $[15,56,57]$. Furthermore, metabolic disorders may associate with low immune function due to damaged function of macrophage and lymphocyte [7, 15, 56-59], which make patients susceptible to disease complications. However, chronic comorbid conditions of COVID-19 patients presents major clinical challenges in terms of diagnosis, ill health, the course of treatment and disease management, which adversely influences treatment choices and outcomes.

In this meta-analysis, the overall pooled crude CFR was $7 \%$ (95\% CI 6-7\%), which was significantly increased with a higher level of chronic comorbid conditions. A recent study estimated the overall CFR for Chinese and Italian patients aged $>48$ years were $6.93 \%$ and $24.8 \%$, respectively [54], which were comparatively higher than our estimate. The distribution of CFR can vary across countries in terms of age-stratified data as well as chronic comorbid conditions [54]. COVID-19 patients who reported no chronic comorbid 
(a) Any type of chronic comorbid condition

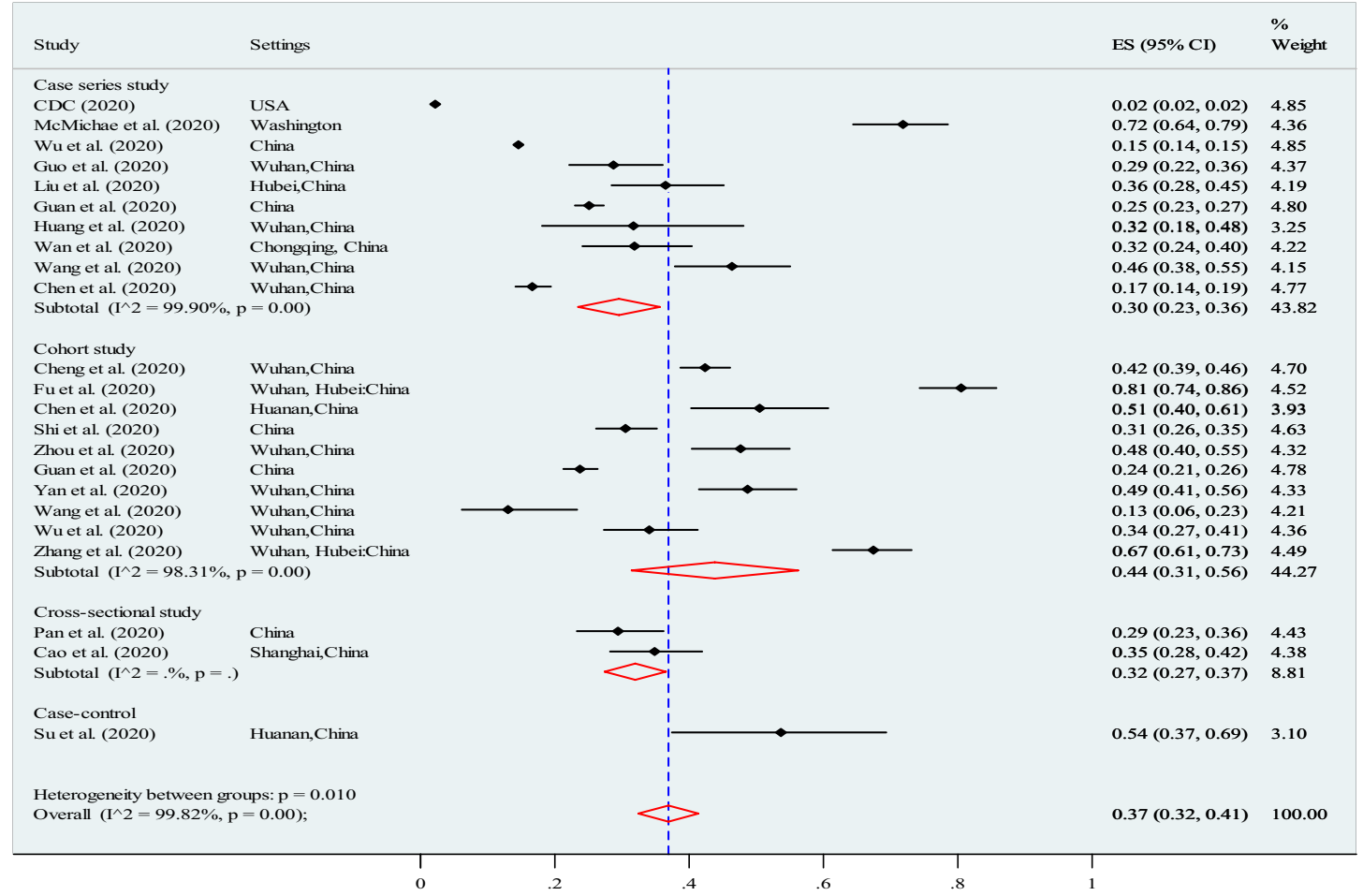

(b) Hypertension

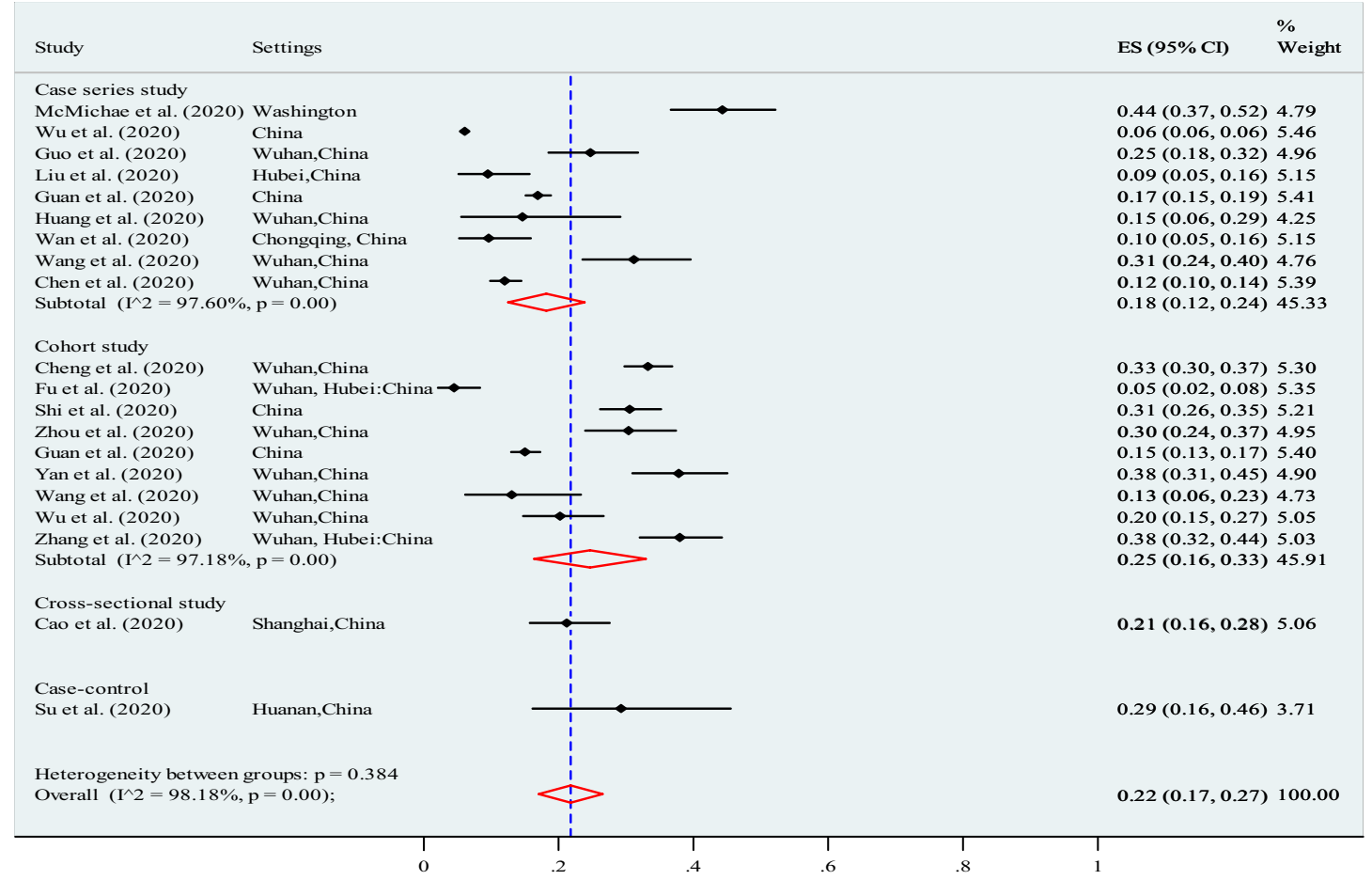

Fig. 2 Meta-analysis of the proportion of comorbidities in COVID-19 infected populations. a Any type of chronic diseases. b Hypertension. c Diabetes. d Cardiovascular disease (CVD). e Respiratory system diseases. f Other chronic diseases 
(c) Diabetes

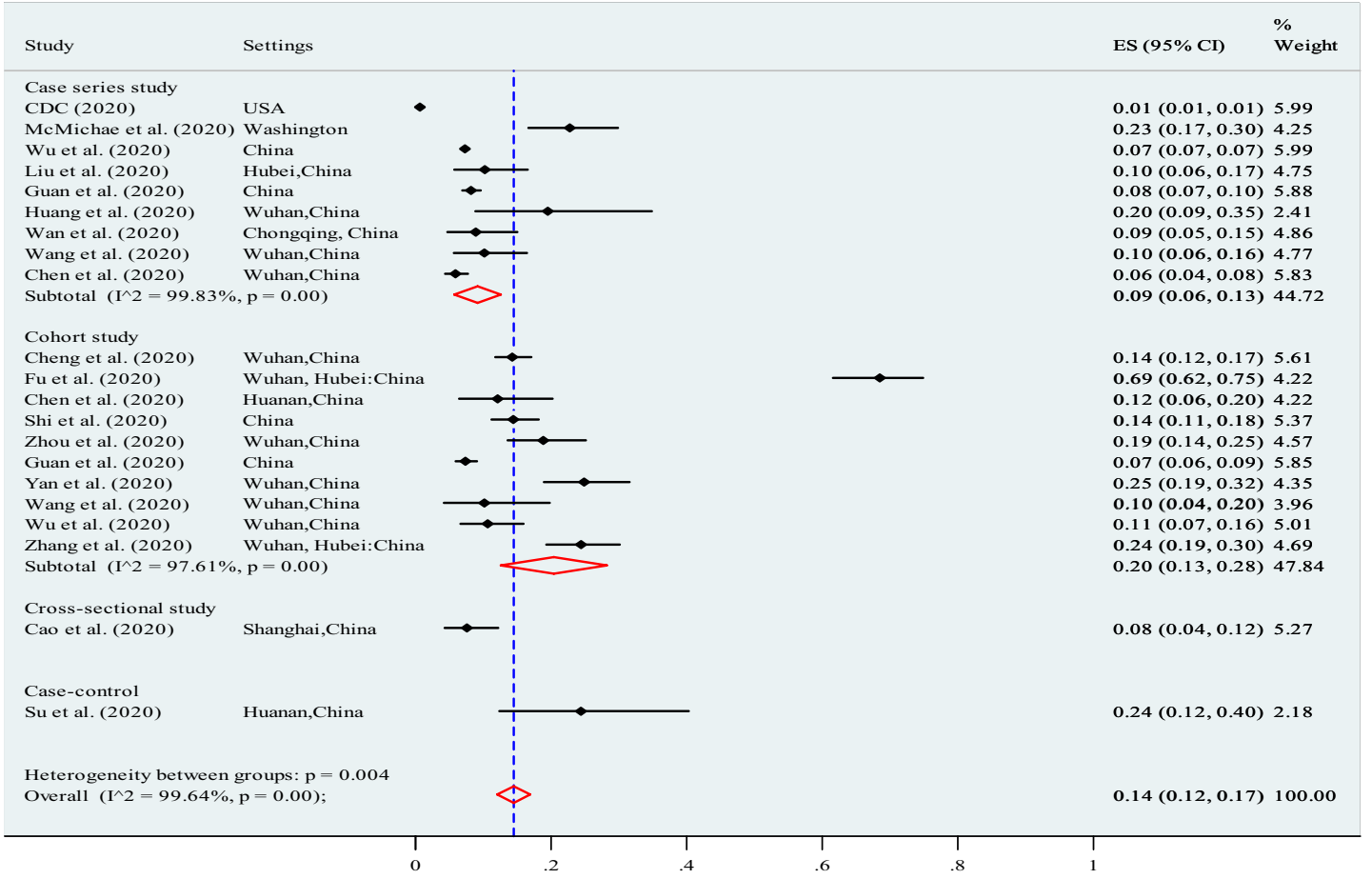

(d) Cardiovascular disease (CVD)

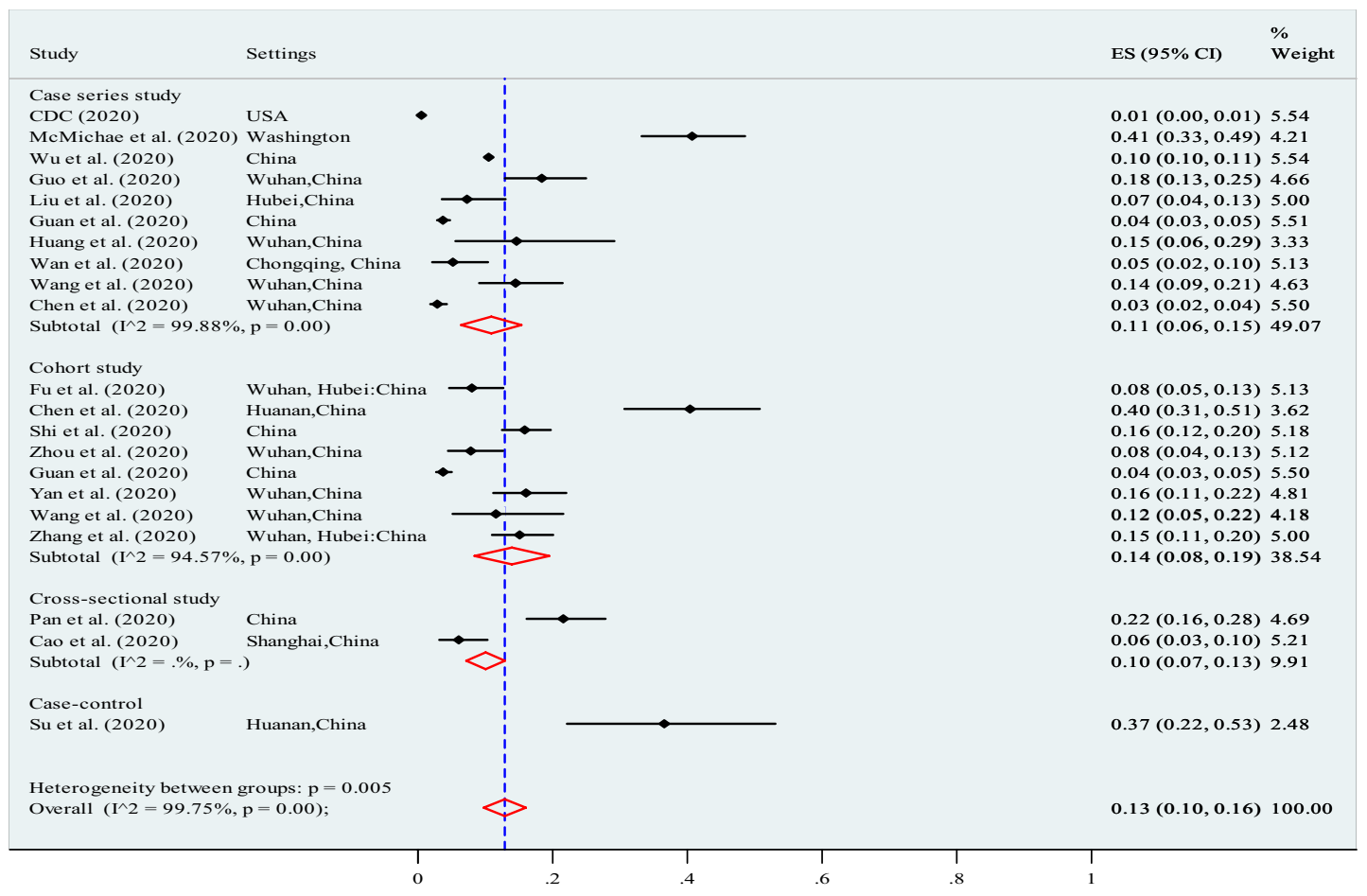

Fig. 2 (continued) 


\section{(e) Respiratory System Disease}

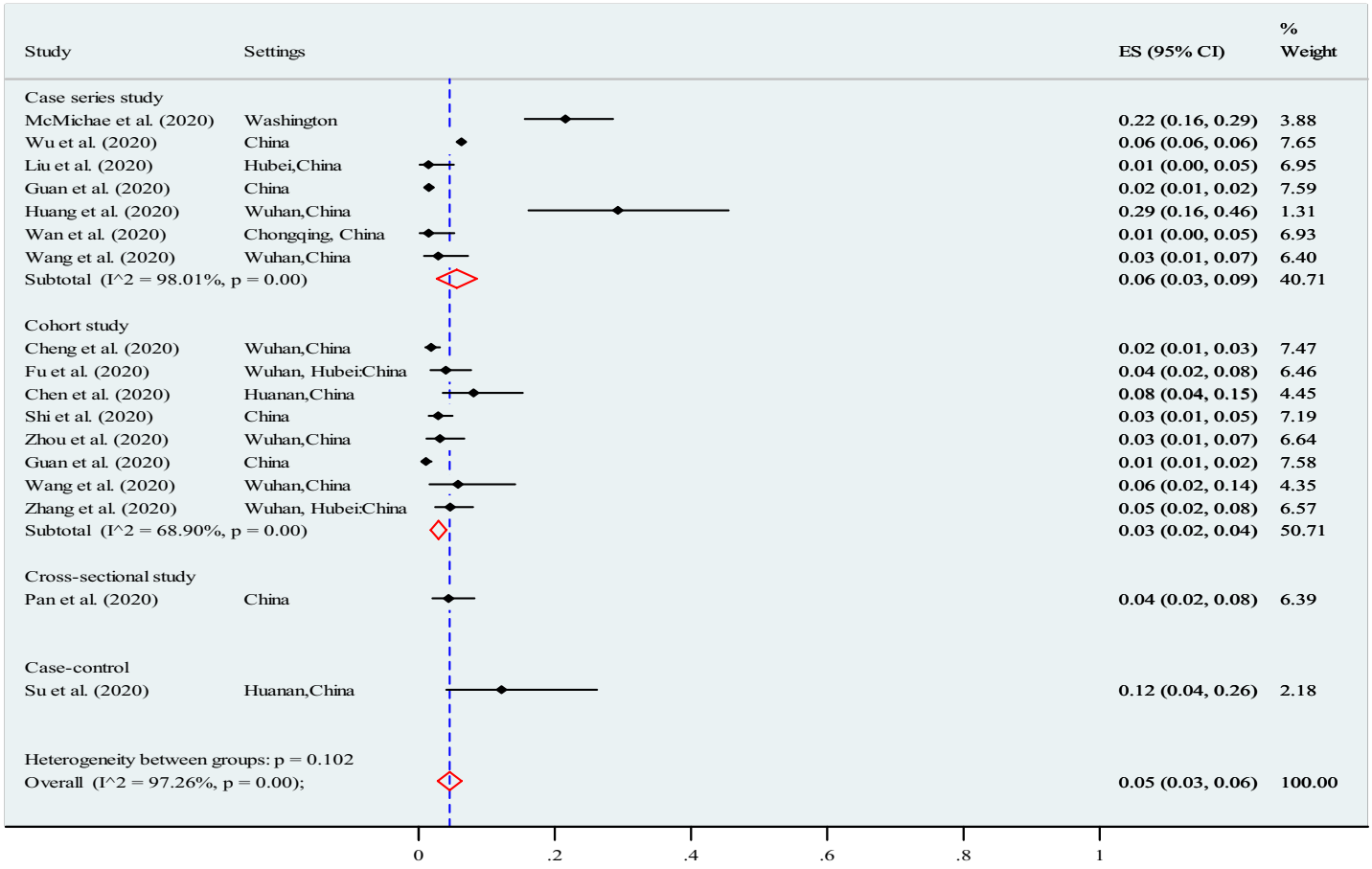

(f) Other chronic diseases

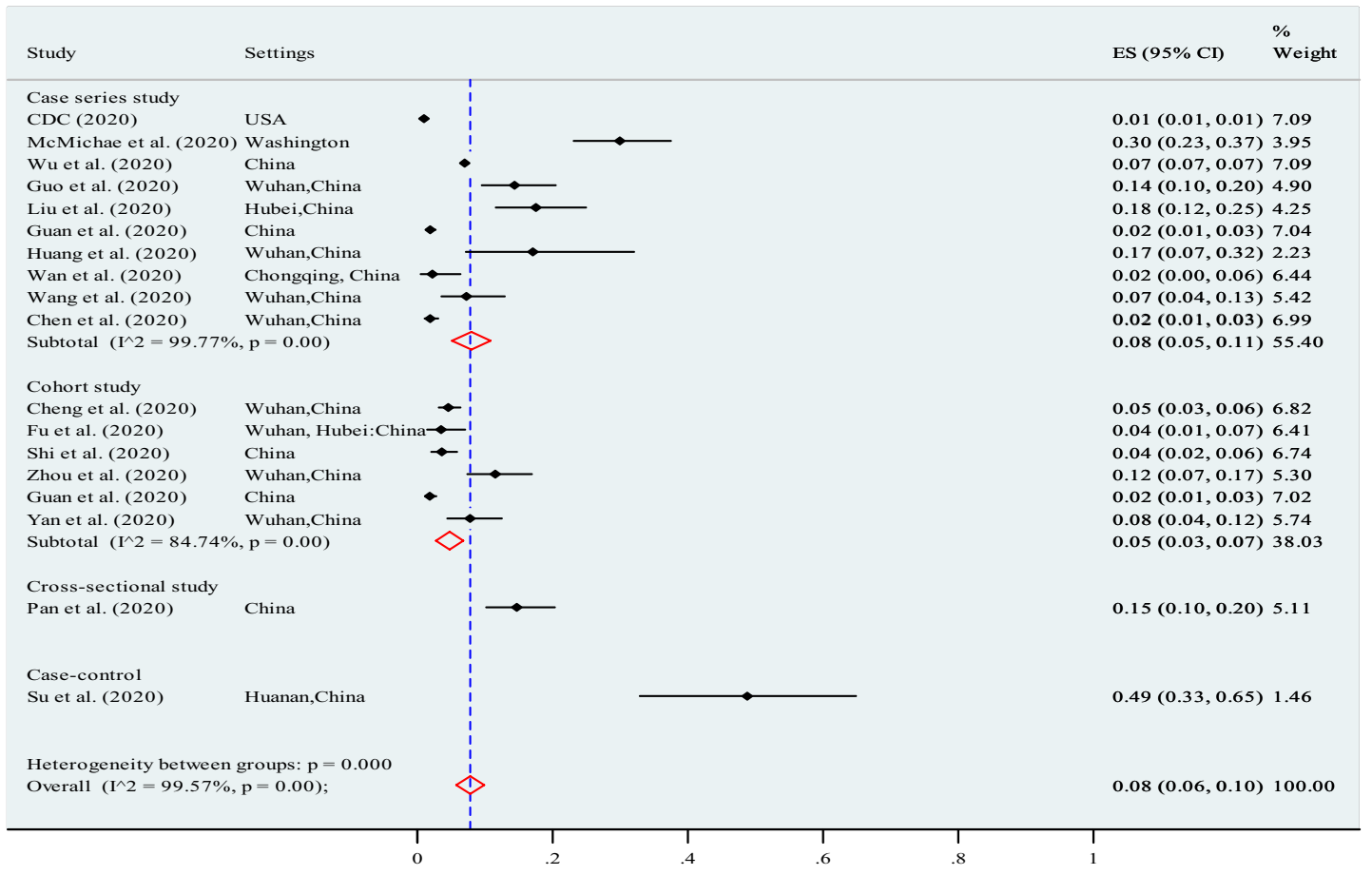

Fig. 2 (continued) 
(a) CFR for at least one chronic comorbid condition

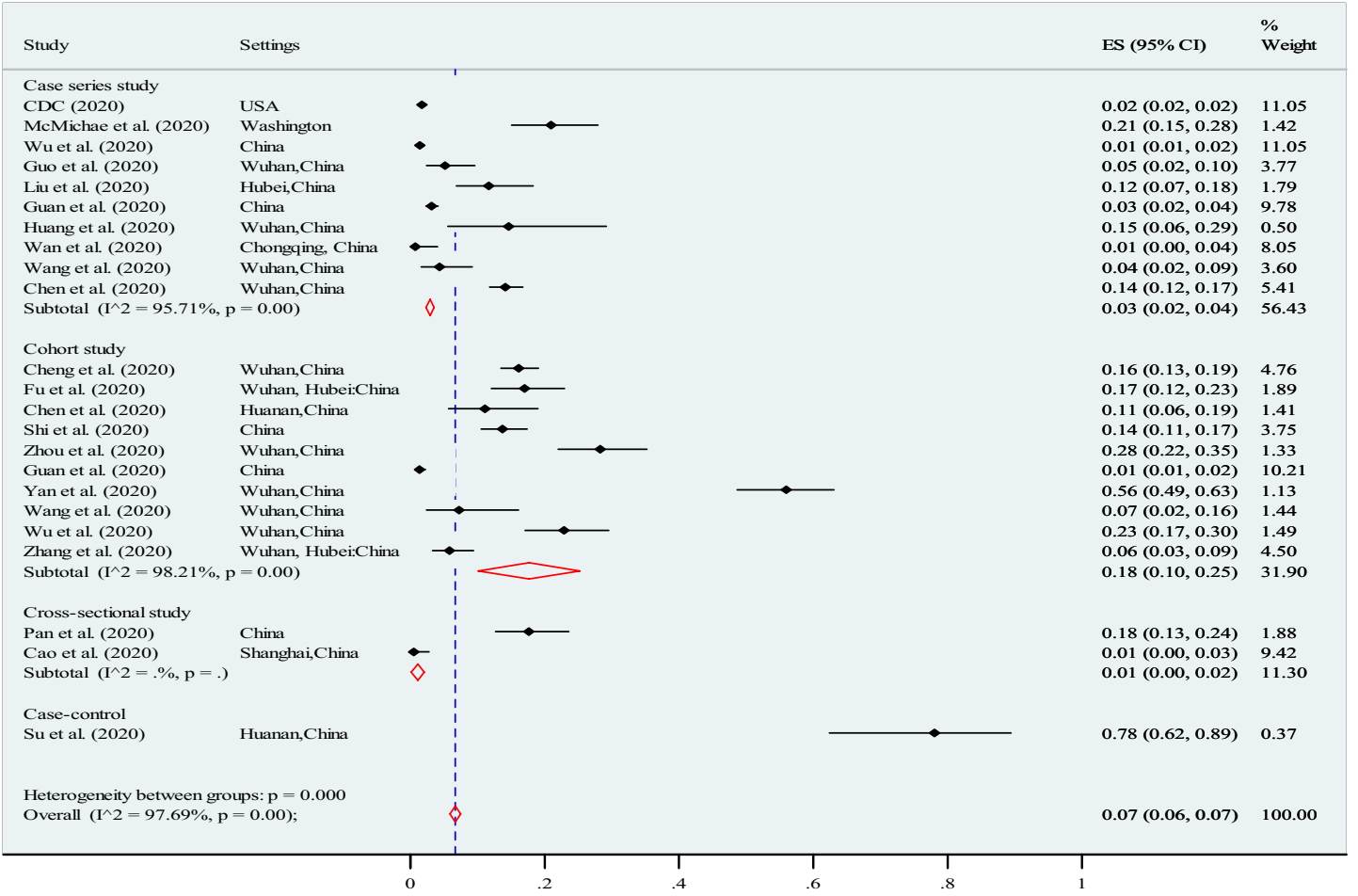

(b) CFR for two chronic comorbid conditions

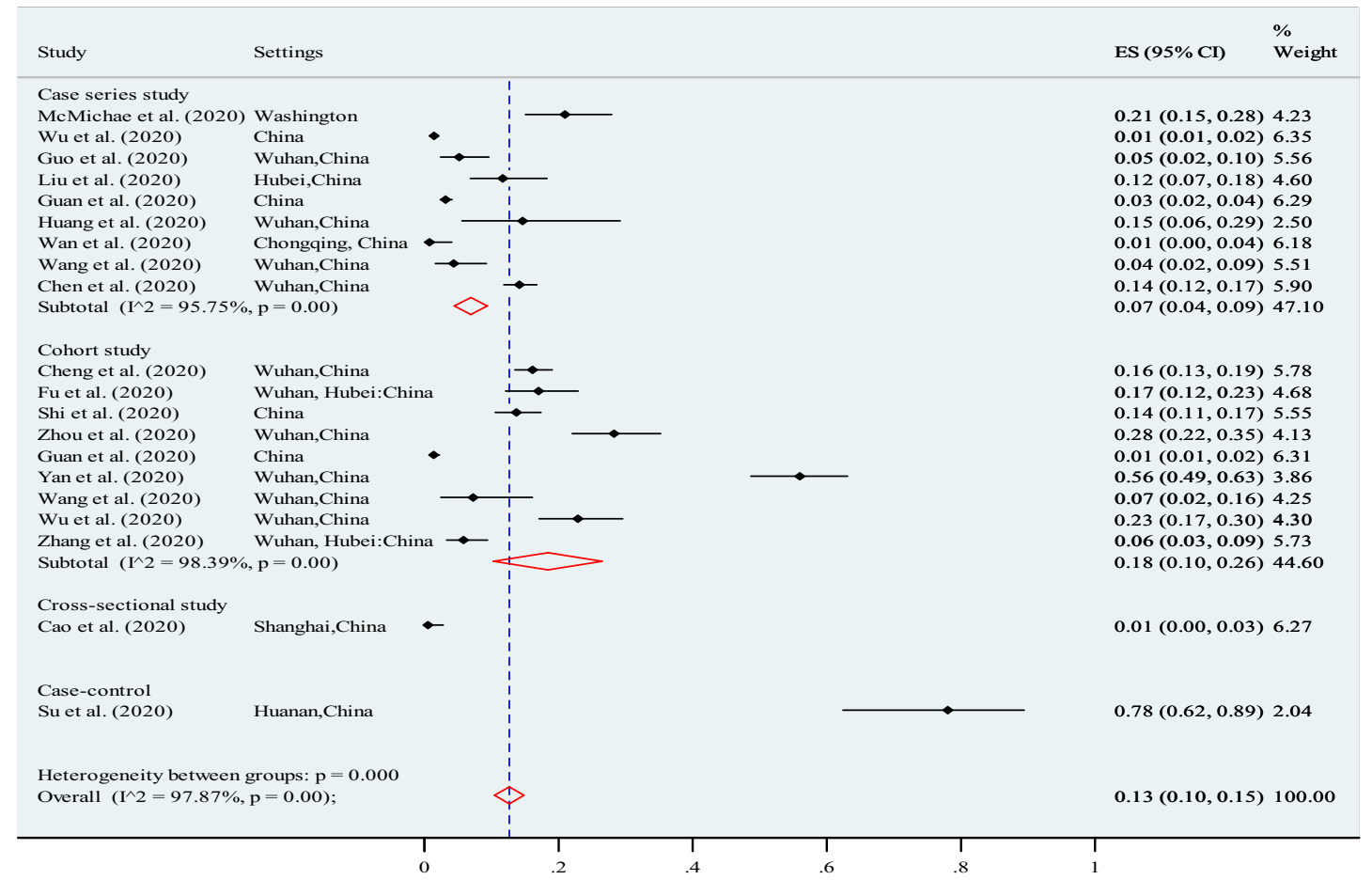

Fig. 3 Association between case fatality ratio (CFR) and number of chronic comorbid conditions in COVID-19 infected populations. a CFR for any type of chronic disease. b CFR for 2 chronic comorbid conditions. c CFR for 3 chronic comorbid conditions. d CFR for 4 chronic comorbid conditions. e CFR for 5 chronic comorbid conditions. $\mathrm{f}$ CFR for 6 or more chronic comorbid conditions 
(c) CFR for three chronic comorbid conditions

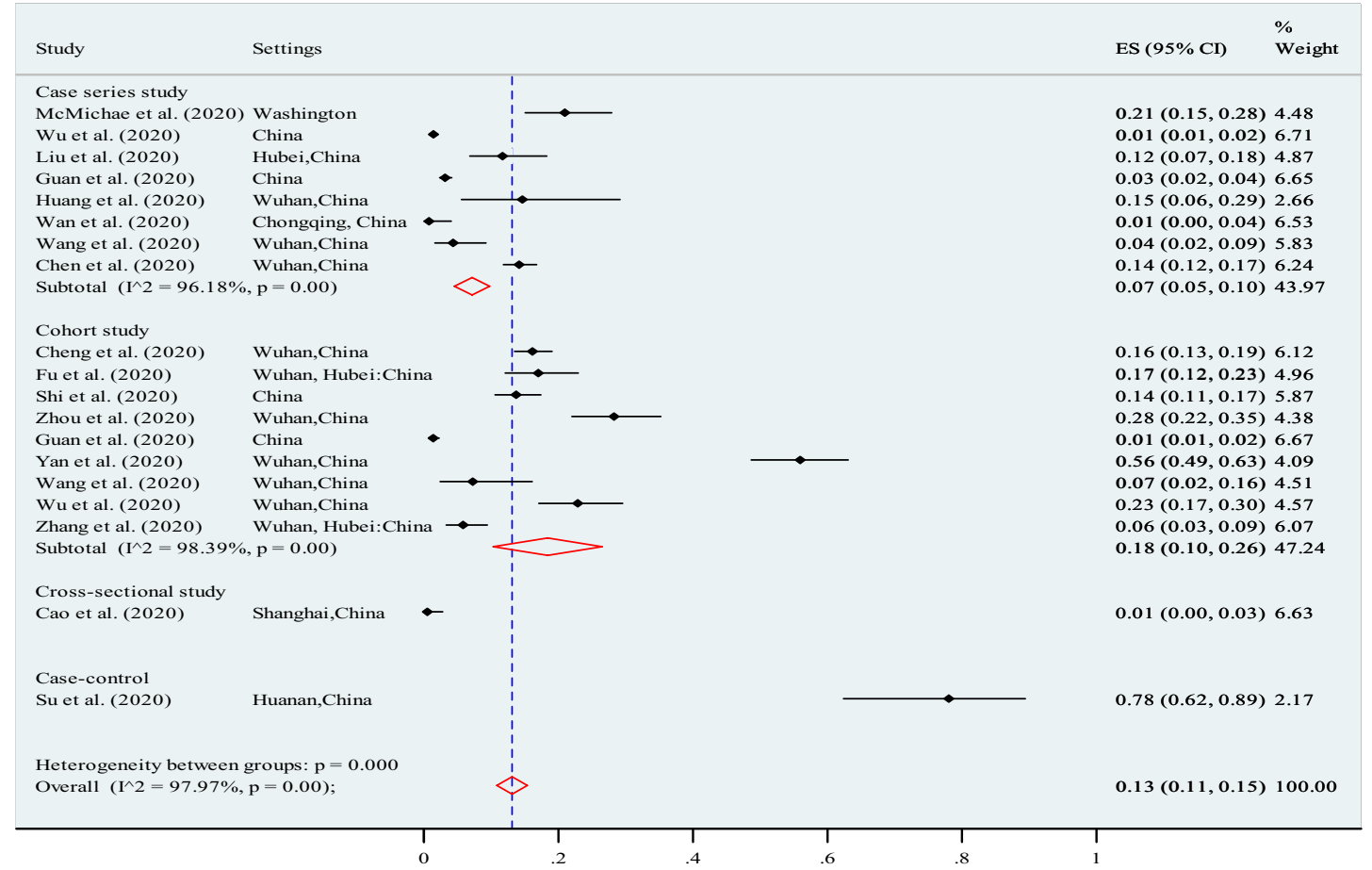

(d) CFR for four chronic comorbid conditions

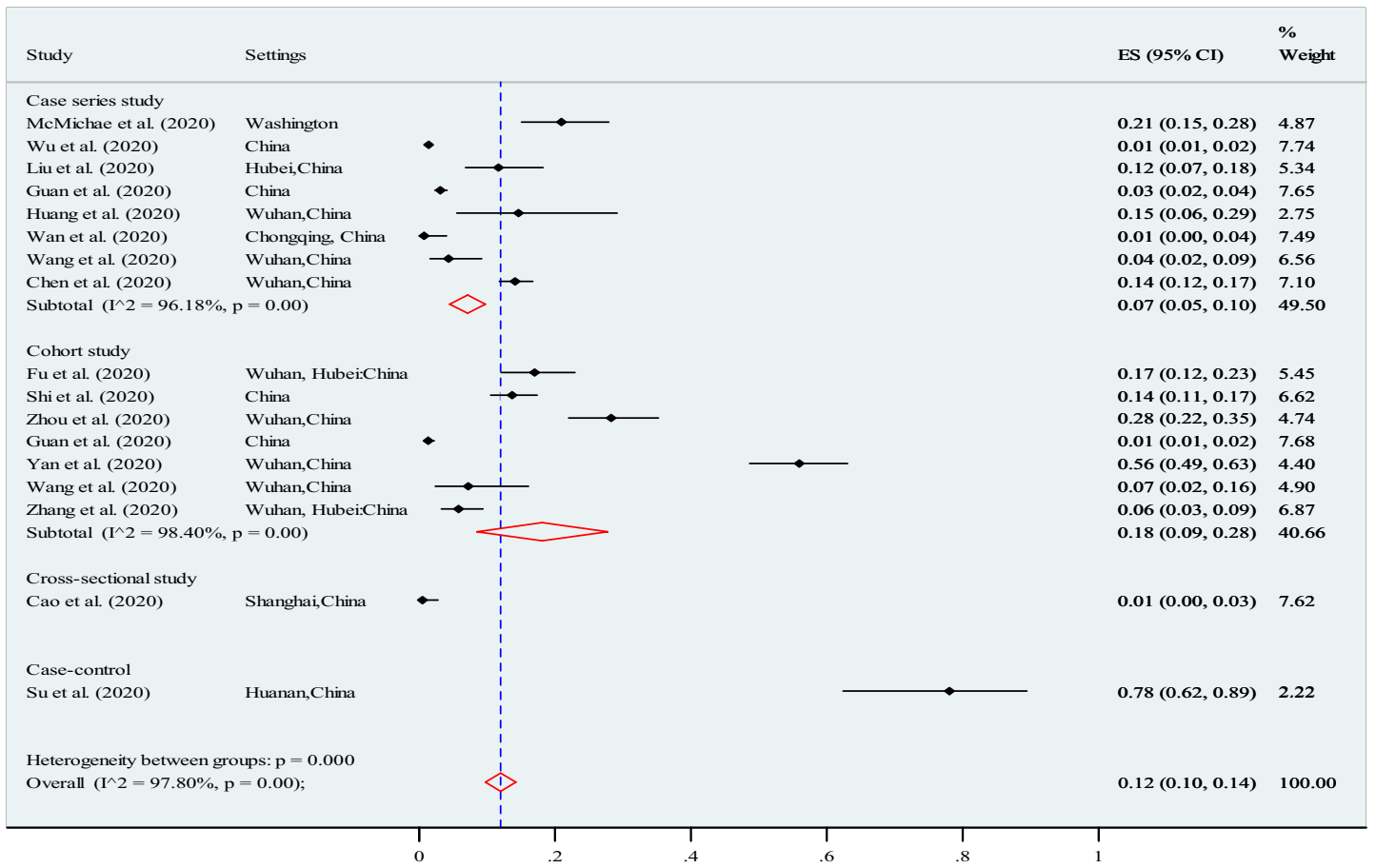

Fig. 3 (continued)

conditions had a lower CFR of $1.4 \%$ compared to patients with comorbid conditions [3]. Some studies suggest that COVID-19 which causes pneumonia may also damage organs [10, 14, 60, 61]. Consequently, patients eventually die of multiple organ failure, shock, acute respiratory distress syndrome, heart failure, arrhythmias, and renal failure 
(e) CFR for five chronic comorbid conditions

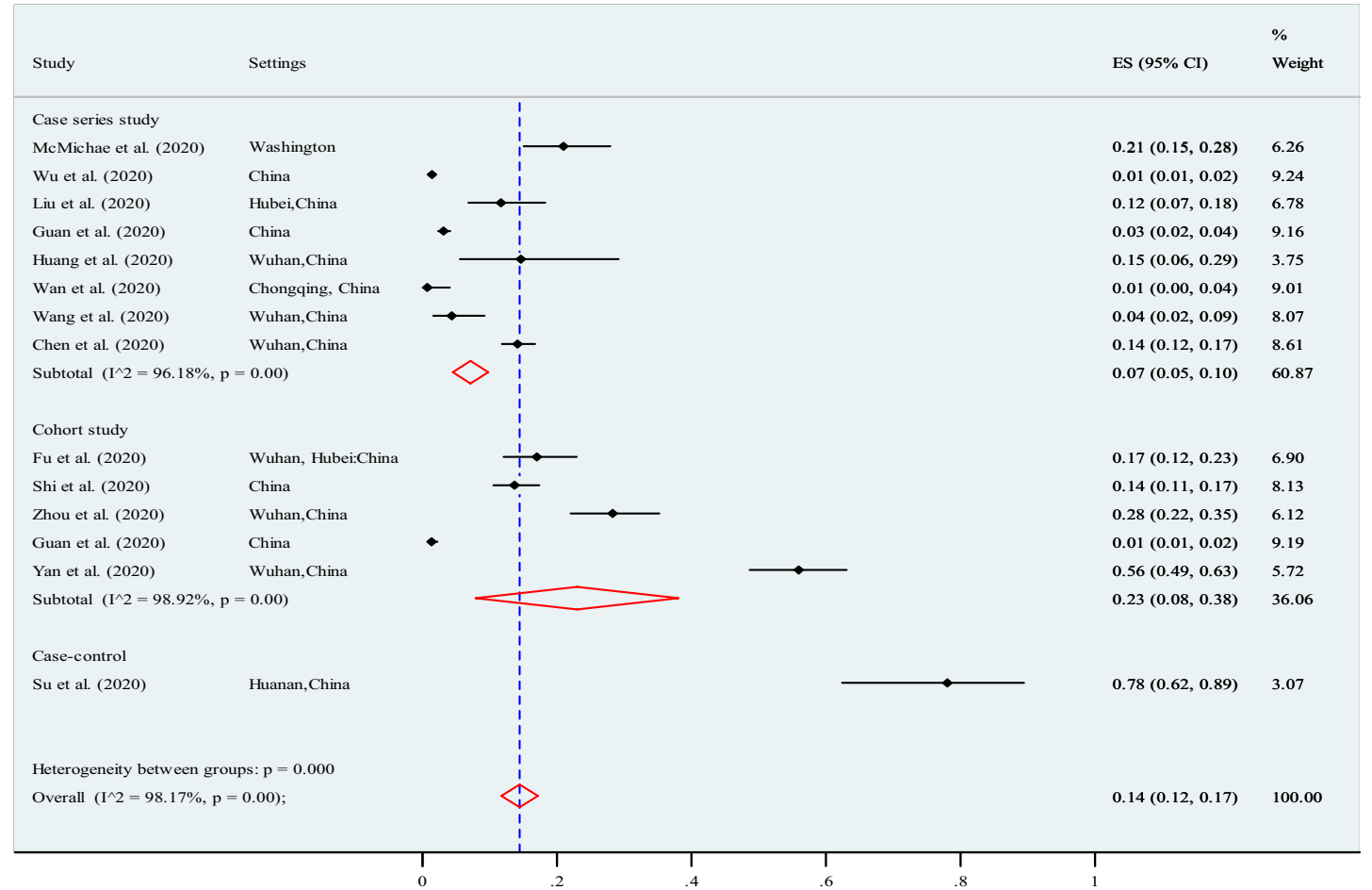

(f) CFR for six or more chronic comorbid conditions

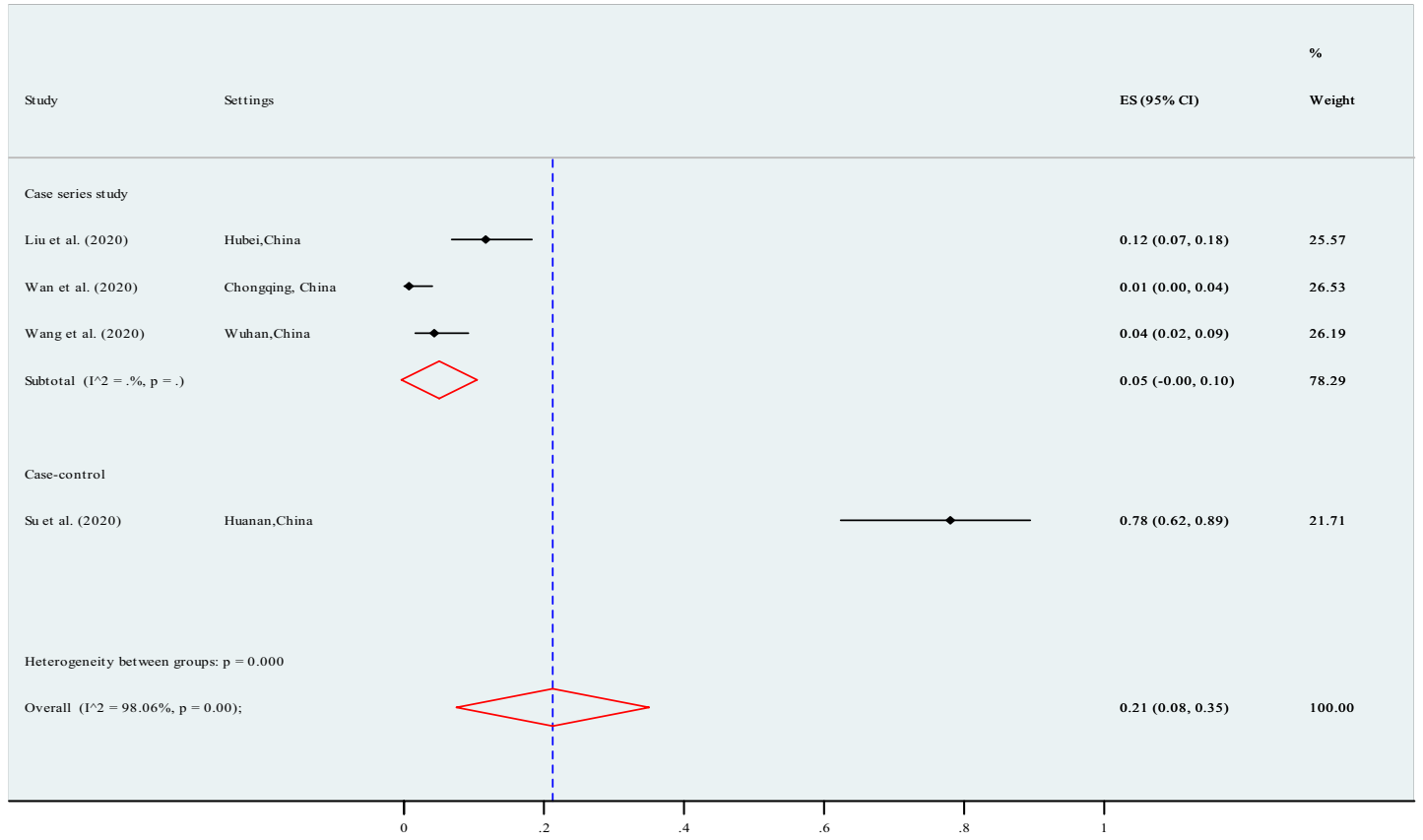

Fig. 3 (continued)

[11-13, 44, 62, 63]. Considering age-stratified data, the CFR of elderly patients ( $\geq$ aged 70 years) was estimated at $37.6 \%$ in Italy and only $11.9 \%$ in China [54]. It can be argued that the elderly patients suffered from more than one underlying chronic comorbid conditions than younger patients $[12,35$, $59,64,65]$. This is similar to findings of a 2013 study of 


\section{(a) CFR for at least 4 reported symptoms}

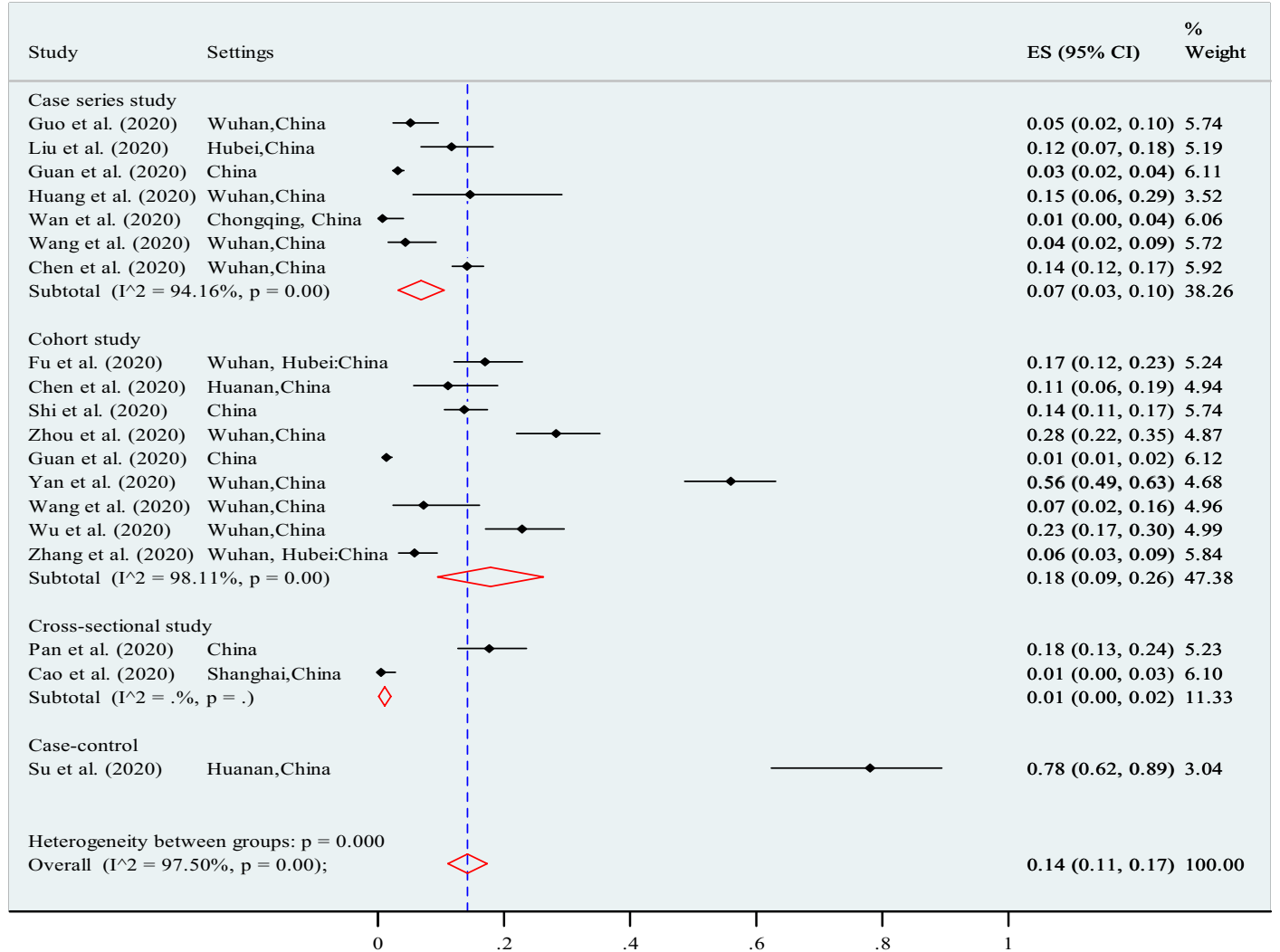

\section{(b) CFR for 5-6 reported symptoms}

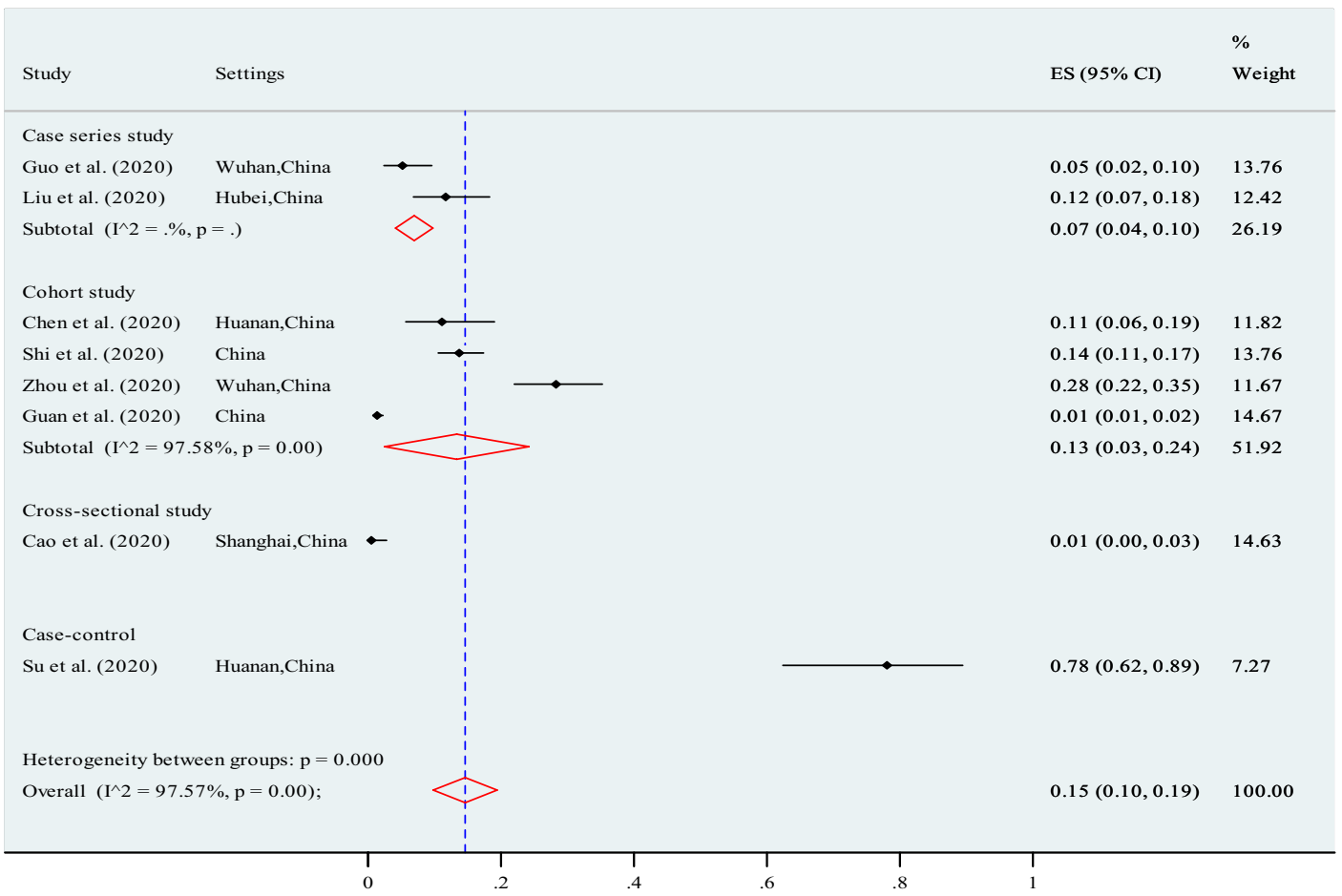

Fig. 4 Association between case fatality ratio (CFR) and reported symptoms in COVID-19 infected populations. a CFR for at least four reported symptoms. b CFR for 5 to 6 reported symptoms. c CFR for 7 to 8 reported symptoms. d CFR for more 8 reported symptoms 
(c) CFR for 7-8 reported symptoms

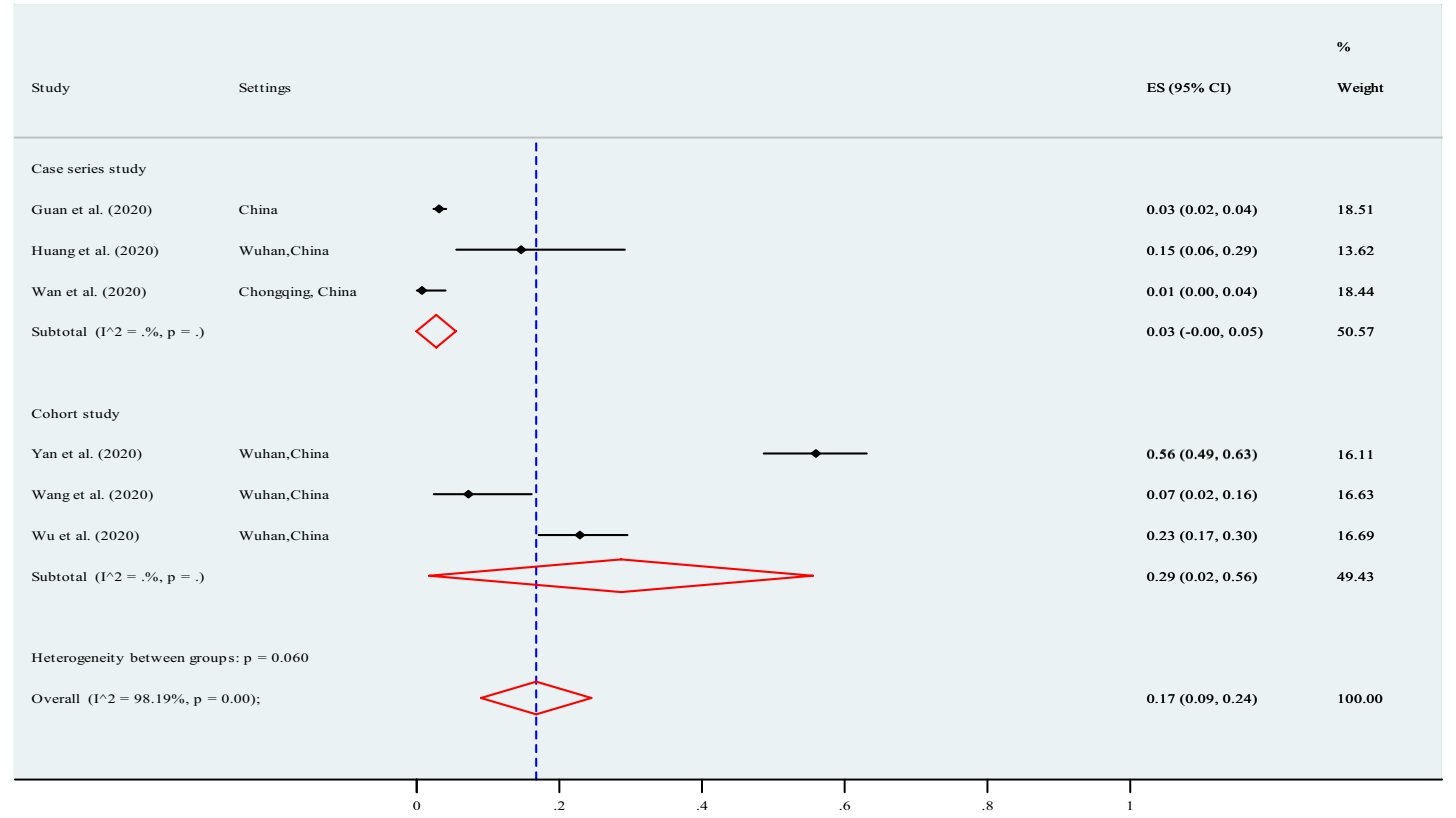

(d) CFR for 9 or more reported symptoms

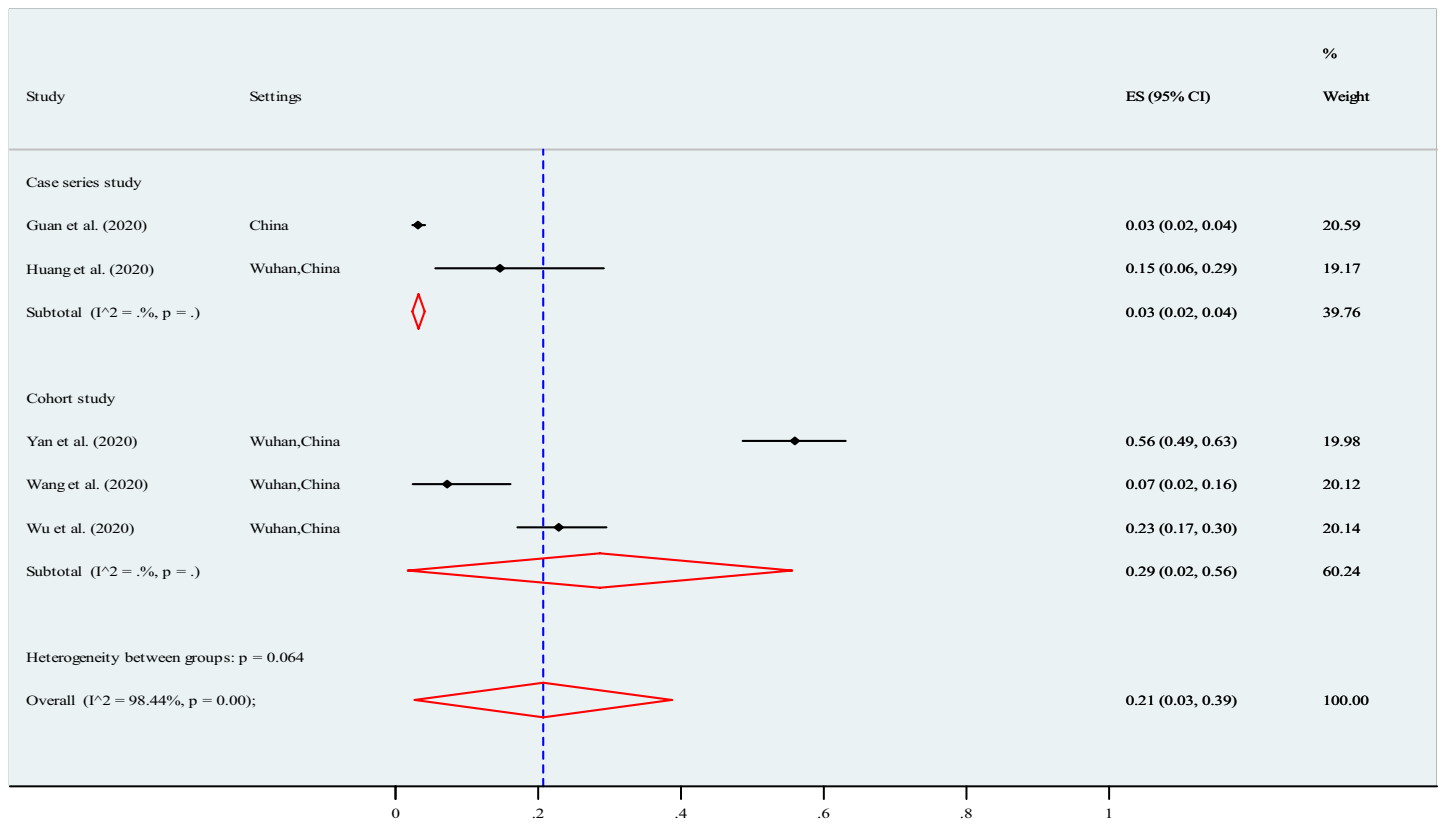

Fig. 4 (continued)

influenza (H7N9) that associated comorbidity among aged people with increased risk of dying [60]. Furthermore, the risk of death among the elderly was aggregated by the low immune system due to multiple medical illnesses $[52,59$, 64, 66-68].

This meta-analysis has some limitations. First, the included studies were heterogeneous in designs, perspectives, and varied in terms of study participants (41 to 122,653$)$. Second, included studies had different followup periods which may be responsible for significant heterogeneity especially because some participant were still hospitalised. Third, few of the included studies considered data on comorbidities of COVID-19 patients. Therefore, it 
Table 3 Stratified analysis of the likelihood of death among COVID-19 infected population

\begin{tabular}{|c|c|c|c|c|}
\hline \multirow[t]{3}{*}{ Characteristics } & \multicolumn{2}{|l|}{ Meta-regression } & \multirow{2}{*}{\multicolumn{2}{|c|}{$\begin{array}{l}\text { Monte Carlo permutation test } \\
\text { for meta-regression }^{\mathrm{a}} \\
p \text { values }\end{array}$}} \\
\hline & \multirow{2}{*}{$\begin{array}{l}\text { Pooled coefficient }(\beta)(95 \% \text { confi- } \\
\text { dence interval, } \mathrm{CI})\end{array}$} & \multirow{2}{*}{$\begin{array}{l}\text { Probability value ( } p \\
\text { value) }\end{array}$} & & \\
\hline & & & Unadjusted & Adjusted \\
\hline \multicolumn{5}{|l|}{ Mean age of total patients } \\
\hline$<50$ years & $0.002(-0.015,0.019)$ & 0.828 & 0.802 & 1.000 \\
\hline$\geq 50$ years & $0.151(0.019,0.321)$ & 0.009 & 0.037 & 0.689 \\
\hline Incubation period in day & $0.011(0.004,0.021)$ & 0.043 & 0.025 & 0.037 \\
\hline \multicolumn{5}{|l|}{ Chronic comorbid conditions } \\
\hline Any type of chronic disease & $0.014(0.005,0.072)$ & 0.007 & 0.026 & 0.017 \\
\hline Hypertension & $0.055(0.013,0.238)$ & 0.054 & 0.968 & 1.000 \\
\hline Diabetes & $0.188(0.130,0.506)$ & 0.023 & 0.017 & 0.033 \\
\hline Cardiovascular disease (CVD) & $0.221(-0.071,0.512)$ & 0.129 & 0.062 & 0.764 \\
\hline Respiratory system disease & $0.331(0.219,0.881)$ & 0.022 & 0.020 & 0.036 \\
\hline Other chronic diseases & $0.346(-0.105,0.796)$ & 0.124 & 0.028 & 0.012 \\
\hline \multicolumn{5}{|l|}{ Number of chronic comorbidity } \\
\hline At least one chronic comorbid condition & $0.014(0.005,0.072)$ & 0.007 & 0.067 & 0.065 \\
\hline 2 chronic comorbid conditions & $0.006(0.001,0.017)$ & 0.026 & 0.058 & 0.046 \\
\hline 3 chronic comorbid conditions & $0.007(0.001,0.017)$ & 0.031 & 0.049 & 0.029 \\
\hline 4 chronic comorbid conditions & $0.009(0.001,0.017)$ & 0.035 & 0.029 & 0.033 \\
\hline 5 chronic comorbid conditions & $0.013(0.001,0.025)$ & 0.048 & 0.055 & 0.010 \\
\hline$\geq 6$ chronic comorbid conditions & $0.012(0.004,0.025)$ & 0.056 & 0.061 & 0.025 \\
\hline \multicolumn{5}{|l|}{ Study design } \\
\hline Case series study & $0.005(-0.012,0.021)$ & 0.687 & 0.780 & 0.920 \\
\hline Case-control study & $0.771(-0.852,2.394)$ & 0.335 & NA & NA \\
\hline Cohort study & $0.016(-0.012,0.043)$ & 0.286 & 0.429 & 0.899 \\
\hline Cross-sectional study & $-0.006(-0.025,0.005)$ & 0.318 & 0.501 & 0.972 \\
\hline \multicolumn{5}{|l|}{ Sample size } \\
\hline$\leq 200$ patients & $-0.01(-0.029,0.009)$ & 0.266 & 0.319 & 0.493 \\
\hline$>200$ patients & $0.011(0.009,0.029)$ & 0.013 & 0.031 & 0.026 \\
\hline Number of studies $(n)$ & 23 & & - & \\
\hline tau-squared $\left(\tau^{2}\right)^{\mathrm{d}}$ & 0.034 & & - & \\
\hline Adjusted $R$-squared $\left(R^{2}\right)^{\mathrm{c}}$ & $45.23 \%$ & & - & \\
\hline$I$-squared $\left(I^{2}\right)^{\mathrm{b}}$ & $96.86 \%$ & & - & \\
\hline Permutations ${ }^{\mathrm{e}}$ & - & & 10,000 & \\
\hline
\end{tabular}

${ }^{a}$ Moment-based estimate of between-study variance without Knapp \& Hartung modification to standard errors

${ }^{\mathrm{b}}$ Percentage $(\%)$ of residual variation due to heterogeneity

${ }^{c}$ Proportion of between-study variance explained with Knapp-Hartung modification

${ }^{\mathrm{d}}$ Residual estimated maximum likelihood estimate of between-study variance

${ }^{\mathrm{e}}$ Monte Carlo methods use random numbers, so results may differ between runs

is possible that overall pooled estimates represent an underestimation due inadequate data on comorbidity.

Our findings provide insight on how public health systems should consider chronic comorbid conditions in the COVID-19 response, the treatment and management of COVID-19 patients. There is an urgent need for protocols on care for patients with comorbid conditions. Symptoms of COVID-19 (e.g., fever, cough or fatigue) are almost similar to those of influenza infections, and to some extent some of the chronic conditions. This calls for urgent action to develop guidelines for differential diagnosis for COVID19 symptoms and comorbidities to enable the public health systems to quickly differentiate the disease from other respiratory system diseases caused by influenza, respiratory syncytial virus, and other respiratory viruses. Considering the insufficient level of ongoing evidence, there is a need to further prioritise research to inform response and risk management decisions, particularly at household level and health 
Table 4 Assessing publication bias

\begin{tabular}{|c|c|c|c|c|c|}
\hline \multirow[t]{2}{*}{ Parameters } & \multirow{2}{*}{$\begin{array}{l}\text { Number } \\
\text { of study }\end{array}$} & \multicolumn{2}{|l|}{ Egger's test $^{\mathrm{a}}$} & \multicolumn{2}{|l|}{ Begg's test $^{\mathrm{b}}$} \\
\hline & & Slope & Bias & Adj. Kendall's score $(P-Q)^{\mathrm{c}}$ & Continuity corrected test \\
\hline \multicolumn{6}{|l|}{ Reported comorbidity } \\
\hline Any type of chronic disease & 23 & $0.03(p=0.004)$ & $14.88(p=0.002)$ & $-95(z=-2.51 ; p=0.012)$ & $z=2.48(p=0.013)$ \\
\hline Hypertension & 20 & $0.05(p<0.001)$ & $6.43(p<0.001)$ & $-04(z=-0.13 ; p=0.897)$ & $z=0.10(p=0.922)$ \\
\hline Diabetes & 21 & $0.01(p=0.042)$ & $8.98(p=0.015)$ & $-54(z=-1.63 ; p=0.103)$ & $z=1.60(p=0.110)$ \\
\hline Cardiovascular disease (CVD) & 21 & $0.01(p=0.132)$ & $8.64(p=0.050)$ & $08(z=0.24 ; p=0.809)$ & $z=0.21(p=0.833)$ \\
\hline Respiratory system disease & 17 & $0.05(p=0.001)$ & $-2.39(p=0.177)$ & $84(z=3.46 ; p=0.001)$ & $z=3.42(p=0.001)$ \\
\hline Other chronic diseases & 18 & $0.01(p=0.001)$ & $6.09(p=0.120)$ & $73(z=2.77 ; p=0.006)$ & $z=2.73(p=0.006)$ \\
\hline \multicolumn{6}{|l|}{ Case fatality rate (CFR) } \\
\hline $\begin{array}{l}\text { At least one chronic comorbid condi- } \\
\text { tion }\end{array}$ & 23 & $0.01(p=0.001)$ & $5.20(p=0.001)$ & $85(z=2.39 ; p=0.025)$ & $z=2.22(p=0.027)$ \\
\hline 2 chronic comorbid conditions & 20 & $0.01(p=0.001)$ & $5.21(p=0.001)$ & $76(z=2.47 ; p=0.014)$ & $z=2.43(p=0.015)$ \\
\hline 3 chronic comorbid conditions & 19 & $0.02(p=0.001)$ & $5.68(p=0.002)$ & $69(z=2.41 ; p=0.016)$ & $z=2.38(p=0.017)$ \\
\hline 4 chronic comorbid conditions & 17 & $0.01(p=0.001)$ & $5.25(p=0.001)$ & $64(z=2.64 ; p=0.008)$ & $z=2.60(p=0.009)$ \\
\hline 5 chronic comorbid conditions & 14 & $0.03(p=0.002)$ & $6.16(p=0.002)$ & $43(z=2.35 ; p=0.019)$ & $z=2.30(p=0.021)$ \\
\hline$\geq 6$ chronic comorbid conditions & 5 & $0.02(p=0.001)$ & $5.68(p=0.002)$ & $69(z=2.41 ; p=0.016)$ & $z=2.38(p=0.017)$ \\
\hline
\end{tabular}

${ }^{a}$ Egger's test for small-study effects was performed in terms of regress standard normal deviate of effect estimate against its standard error

${ }^{b}$ Begg's test was performed to detect publication bias for small-study effects

${ }^{c}$ Rank correlation between standardized effect estimate and its standard error

care facilities. Furthermore, there is a need to investigate the association of household and health facilities care among COVID-19 patients to reduce stigmatisation. Anecdotal evidence suggest that people discharged from government quarantine or after recovering in hospitals are subjected to communal violence and chased away from their homes by their neighbours. Enhancing the systematic and real-time sharing of epidemiologic data, clinical outcomes and experience is critical to inform the global response.

\section{Conclusions}

Chronic comorbid conditions (e.g., hypertension, diabetes mellitus, cardiovascular disease, respiratory disease, and other chronic diseases) were identified as high risk factors. Considering the insufficient level of evidence, there is need to further prioritise research to inform response and risk management decisions, particularly at household level and health care facilities. Furthermore, there is need to investigate the association of household and health facilities care among COVID-19 patients. We call on countries with the greatest knowledge on COVID-19 to further enhance the systematic and real-time sharing of epidemiologic data, clinical outcomes and experience to inform the global response. Therefore, targeted public health vaccination interventions might be adopted by developing herd immune system to save people with chronic conditions from COVID-19 and other associated respiratory infections.
Acknowledgements This research paper was drafted and completed during the first author's postdoctoral research work at the School of Social Sciences, Western Sydney University, New South Wales, Australia. We would like to gratefully acknowledge the study's participants, researchers, reviewers and the academic editors of our manuscript.

Author contributions RAM and AMNR conceptualised the study. RAM led the literature review with contributions from AMNR and JKK. RAM led the data analysis with contributions from AMNR. RAM, AMNR and JKK led the data interpretation. RAM wrote the first draft of the manuscript with input from AMNR and JKK. All named authors contributed to development of the analysis plan, collection and analysis of primary data, data interpretation, and critically reviewed the revised initial draft of the manuscript. All authors read and approved the final draft.

Funding This study was conducted without financial support from any funding body.

\section{Compliance with ethical standards}

Conflict of interest The authors declared no conflicts of interest in this work.

Ethical approval The study does not require ethical approval, because the meta-analysis is based on published research and the original data are anonymous. All of the patient information was de-identified.

\section{Appendix}

See Tables 5, 6, 7 and 8. 
Table 5 Quality score assessment for cohort study

\begin{tabular}{|c|c|c|c|c|c|c|c|c|c|c|c|c|c|}
\hline \multirow[t]{2}{*}{ Studies } & \multicolumn{11}{|c|}{ Quality assessment indicators } & \multirow[t]{2}{*}{ Overall score } & \multirow[t]{2}{*}{ Overall appraisal } \\
\hline & $\mathrm{Q}_{1}$ & $\mathrm{Q}_{2}$ & $\mathrm{Q}_{3}$ & $\mathrm{Q}_{4}$ & $\mathrm{Q}_{5}$ & $\mathrm{Q}_{6}$ & $\mathrm{Q}_{7}$ & $\mathrm{Q}_{8}$ & $\mathrm{Q}_{9}$ & $\mathrm{Q}_{10}$ & $\mathrm{Q}_{11}$ & & \\
\hline Wang et al. $[5,41]$ & $\checkmark$ & $\checkmark$ & $\checkmark$ & $\checkmark$ & $\checkmark$ & $x$ & $\checkmark$ & $\checkmark$ & $x$ & $x$ & $\checkmark$ & 8 (medium) & Included \\
\hline Guan et al. $[39,44]$ & $\checkmark$ & $\checkmark$ & $\checkmark$ & $\checkmark$ & $\checkmark$ & $x$ & $\checkmark$ & $x$ & $x$ & ${ }^{\circledR}$ & $\checkmark$ & 7.5 (medium) & Included \\
\hline Zhang et al. [12] & $\checkmark$ & $\checkmark$ & $\checkmark$ & $\checkmark$ & $\checkmark$ & $x$ & $\checkmark$ & $\checkmark$ & $\checkmark$ & X & $\checkmark$ & 9 (high) & Included \\
\hline Chen et al. $[6,45]$ & $\checkmark$ & $\checkmark$ & $\checkmark$ & $\checkmark$ & $x$ & $x$ & $\checkmark$ & $x$ & $x$ & ${ }^{\circledR}$ & $\checkmark$ & 6.5 (medium) & Included \\
\hline Zhou et al. [34] & $\checkmark$ & $\checkmark$ & $\checkmark$ & $\checkmark$ & $x$ & $x$ & $\checkmark$ & $\checkmark$ & $x$ & $x$ & $\checkmark$ & 7 (medium) & Included \\
\hline Cheng et al. [36] & $\checkmark$ & $\checkmark$ & $\checkmark$ & $\checkmark$ & $x$ & $x$ & $\checkmark$ & $x$ & $x$ & (B) & $\checkmark$ & 6.5 (medium) & Included \\
\hline Fu et al. [37] & $\checkmark$ & $\checkmark$ & $\checkmark$ & $\checkmark$ & $\checkmark$ & $x$ & $\checkmark$ & $x$ & $x$ & (®) & $\checkmark$ & 7.5 (medium) & Included \\
\hline Wu et al. $[35,47]$ & $\checkmark$ & $\checkmark$ & $\checkmark$ & $\checkmark$ & $\checkmark$ & $x$ & $\checkmark$ & $\checkmark$ & $x$ & $x$ & $\checkmark$ & 8.5 (medium) & Included \\
\hline Yan et al. [40] & $\checkmark$ & $\checkmark$ & $\checkmark$ & $\checkmark$ & $x$ & $x$ & $\checkmark$ & ${ }^{\circledR}$ & $x$ & $x$ & $\checkmark$ & 6.5 (medium) & Included \\
\hline Shi et al. [38] & $\checkmark$ & $\checkmark$ & $\checkmark$ & $\checkmark$ & $x$ & $\times$ & $\checkmark$ & $\checkmark$ & $\checkmark$ & ${ }^{\circledR}$ & $\checkmark$ & 8.5 (medium) & Included \\
\hline
\end{tabular}

Note $\checkmark=$ yes $(=1), X=$ no $(=0),{ }^{\circledR}=$ unclear $(=0.5)$; quality assessment decision rules for 11 scales [(i) poor if the overall score $\leq 5$, (ii) medium if overall scores is 6 to 9 , and (iii) high if the overall score $>9$ ], $\mathrm{Q}_{1}$. Were the two groups similar and recruited from the same population? $\mathrm{Q}_{2}$. Were the exposures measured similarly to assign people to both exposed and unexposed groups? $\mathrm{Q}_{3}$. Was the exposure measured in a valid and reliable way? $\mathrm{Q}_{4}$. Were confounding factors identified? $\mathrm{Q}_{5}$. Were strategies to deal with confounding factors stated? $\mathrm{Q}_{6}$. Were the groups/participants free of the outcome at the start of the study (or at the moment of exposure)? $\mathrm{Q}_{7}$. Were the outcomes measured in a valid and reliable way? $\mathrm{Q}_{8}$. Was the follow-up time reported and sufficient to be long enough for outcomes to occur? $\mathrm{Q}_{9}$. Was follow-up complete, and if not, were the reasons to loss to follow-up described and explored? $\mathrm{Q}_{10}$. Were strategies to address incomplete follow-up utilized? $\mathrm{Q}_{11}$. Was appropriate statistical analysis used?

Table 6 Quality score assessment for cross-sectional study

\begin{tabular}{lll}
\hline Quality assessment indicators & Selected studies & Pan et al. [42] \\
\cline { 2 - 3 } & Cao et al. [43] & $\checkmark$ \\
Q1. Were the criteria for inclusion in the sample clearly defined? & $\checkmark$ & $\checkmark$ \\
Q2. Were the study subjects and the setting described in detail? & $\checkmark$ & $\checkmark$ \\
Q3. Was the exposure measured in a valid and reliable way? & $\checkmark$ & $\checkmark$ \\
Q4. Were objective, standard criteria used for measurement of the condition? & $\times$ & $\checkmark$ \\
Q5. Were confounding factors identified? & $\checkmark$ & $\checkmark$ \\
Q6. Were strategies to deal with confounding factors stated? & $\checkmark$ & $\checkmark$ \\
Q7. Were the outcomes measured in a valid and reliable way? & (high) \\
Q8. Was appropriate statistical analysis used? & Included \\
Overall quality score & Included
\end{tabular}

Note $\checkmark=$ yes $(=1), X=$ no $(=0),{ }^{\circledR}=$ unclear $(=0.5)$; quality assessment decision rules for 8 scales: (i) poor if the overall score $<5$, (ii) medium if overall scores is 5 to 6 , and (iii) high if the overall scores $>6$ 
Table 7 Quality score assessment for case-series study

\begin{tabular}{|c|c|c|c|c|c|c|c|c|c|c|c|c|}
\hline \multirow[t]{2}{*}{ Study } & \multicolumn{10}{|c|}{ Quality assessment indicators } & \multirow[t]{2}{*}{ Overall scores } & \multirow[t]{2}{*}{ Overall appraisal } \\
\hline & $\mathrm{Q}_{1}$ & $\mathrm{Q}_{2}$ & $\mathrm{Q}_{3}$ & $\mathrm{Q}_{4}$ & $\mathrm{Q}_{5}$ & $\mathrm{Q}_{6}$ & $\mathrm{Q}_{7}$ & $\mathrm{Q}_{8}$ & $\mathrm{Q}_{9}$ & $\mathrm{Q}_{10}$ & & \\
\hline Chen et al. $[6,45]$ & $\checkmark$ & $\checkmark$ & $\checkmark$ & $\checkmark$ & $\checkmark$ & $\checkmark$ & $\checkmark$ & $\checkmark$ & $\checkmark$ & $\checkmark$ & 10 (high) & Included \\
\hline Guan et al. $[39,44]$ & $\checkmark$ & $\checkmark$ & $\checkmark$ & $\checkmark$ & $\checkmark$ & $\checkmark$ & $\checkmark$ & $\checkmark$ & $\checkmark$ & $\checkmark$ & 10 (high) & Included \\
\hline Guo et al. [48] & $\checkmark$ & $\checkmark$ & (®) & $\checkmark$ & $\checkmark$ & $\checkmark$ & $\checkmark$ & $\checkmark$ & $\checkmark$ & $x$ & 8.5 (high) & Included \\
\hline Huang et al. [7] & $\checkmark$ & $\checkmark$ & $\checkmark$ & $\checkmark$ & $\checkmark$ & $\checkmark$ & $\checkmark$ & $\checkmark$ & $\checkmark$ & $\checkmark$ & 10 (high) & Included \\
\hline McMichael et al. [2] & $\checkmark$ & $\checkmark$ & $\checkmark$ & $\checkmark$ & $\checkmark$ & $\checkmark$ & $\checkmark$ & $\checkmark$ & $x$ & $x$ & 8 (medium) & Included \\
\hline Wu et al. [35, 47] & $\checkmark$ & $\checkmark$ & $\checkmark$ & $x$ & $x$ & $\checkmark$ & $x$ & $\checkmark$ & $\checkmark$ & $x$ & 6 (medium) & Included \\
\hline Wan et al. [49] & $\checkmark$ & $\checkmark$ & $\checkmark$ & $\checkmark$ & $\checkmark$ & $\checkmark$ & $\checkmark$ & $\checkmark$ & $\checkmark$ & $\checkmark$ & 10 (high) & Included \\
\hline Wang et al. $[5,41]$ & $\checkmark$ & $\checkmark$ & $\checkmark$ & $\checkmark$ & $\checkmark$ & $\checkmark$ & $\checkmark$ & $\checkmark$ & $\checkmark$ & $\checkmark$ & 10 (high) & Included \\
\hline CDC [8] & $\checkmark$ & $\checkmark$ & $\checkmark$ & $\checkmark$ & $\checkmark$ & $\checkmark$ & $\checkmark$ & $\checkmark$ & (B) & $x$ & 8.5 (high) & Included \\
\hline Liu et al. [46] & $\checkmark$ & $\checkmark$ & $\checkmark$ & $\checkmark$ & $\checkmark$ & $\checkmark$ & $\checkmark$ & $x$ & $\checkmark$ & $\checkmark$ & 9 (high) & Included \\
\hline
\end{tabular}

Note $\checkmark=$ yes $(=1), X=$ no $(=0),{ }^{\circledR}=\operatorname{unclear}(=0.5)$; quality assessment decision rules for 10 scales: (i) poor if the overall scores $<6$, (ii) medium if overall scores is 6 to 8 , and (iii) high if the overall scores $>8$. $\mathrm{Q}_{1}$. Were there clear criteria for inclusion in the case series? $\mathrm{Q}_{2}$. Was the condition measured in a standard, reliable way for all participants included in the case series? $\mathrm{Q}_{3}$. Were valid methods used for identification of the condition for all participants included in the case series? $\mathrm{Q}_{4}$. Did the case series have consecutive inclusion of participants? $\mathrm{Q}_{5}$. Did the case series have complete inclusion of participants? $\mathrm{Q}_{6}$. Was there clear reporting of the demographics of the participants in the study? $\mathrm{Q}_{7}$. Was there clear reporting of clinical information of the participants? $\mathrm{Q}_{8}$. Were the outcomes or follow-up results of cases clearly reported? $\mathrm{Q}_{9}$. Was there clear reporting of the presenting site(s)/clinic(s) demographic information? $\mathrm{Q}_{10}$. Was statistical analysis appropriate?

\begin{tabular}{ll}
\hline Quality assessment indicators & Su et al. [50] \\
\hline $\begin{array}{l}\text { Q1. Were the groups comparable other than the presence of disease in cases or the absence } \\
\text { of disease }\end{array}$ & $\checkmark$ \\
in controls? & \\
Q2. Were cases and controls matched appropriately? & $\checkmark$ \\
Q3. Were the same criteria used for identification of cases and controls? & $\checkmark$ \\
Q4. Was exposure measured in a standard, valid and reliable way? & $\checkmark$ \\
Q5. Was exposure measured in the same way for cases and controls? & $\checkmark$ \\
Q6. Were confounding factors identified? & $\checkmark$ \\
Q7. Were strategies to deal with confounding factors stated? & $\checkmark$ \\
Q8. Were outcomes assessed in a standard, valid and reliable way for cases and controls? \\
Q9. Was the exposure period of interest long enough to be meaningful? \\
Q10. Was appropriate statistical analysis used? & $\checkmark$ \\
Overall quality score & 8 (medium) \\
Overall appraisal & Included \\
\hline
\end{tabular}

Note $\checkmark=$ yes $(=1), X=$ no $(=0),{ }^{\circledR}=$ unclear $(=0.5)$; quality assessment decision rules for 10 scales: (i) poor if the overall scores $<6$, (ii) medium if the overall scores is 6 to 8 , and (iii) high if the overall scores $>8$

\section{References}

1. World Health Organization (WHO). Coronavirus (COVID-19). 2020. https://who.sprinklr.com/. Accessed 9 Apr 2020.

2. McMichael TM, Currie DW, Clark S, Pogosjans S, Kay M, Schwartz NG, et al. Epidemiology of Covid-19 in a longterm care facility in King County, Washington. N Engl J Med. 2020;382:2005-2011. https://doi.org/10.1056/NEJMoa2005412.

3. World Health Organization (WHO). Report of the WHO-China joint mission on coronavirus disease 2019 (COVID-19). 2020.
4. European Centre for Disease Prevention and Control. Novel coronavirus disease 2019 (COVID-19) pandemic: increased transmission in the EU/EEA and the UK-sixth update-12 March 2020. Stockholm: ECDC; 2020.

5. Wang D, Hu B, Hu C, Zhu F, Liu X, Zhang J, et al. Clinical characteristics of 138 hospitalized patients with 2019 novel coronavirus-infected pneumonia in Wuhan, China. JAMA. 2020;323:1061-9.

6. Chen N, Zhou M, Dong X, Qu J, Gong F, Han Y, et al. Epidemiological and clinical characteristics of 99 cases of 2019 novel coronavirus pneumonia in Wuhan, China: a descriptive study. Lancet. 
2020;395:507-13. https://doi.org/10.1016/S0140-6736(20)30211 $-7$.

7. Huang C, Wang Y, Li X, Ren L, Zhao J, Hu Y, et al. Clinical features of patients infected with 2019 novel coronavirus in Wuhan, China. Lancet. 2020;395:497-506.

8. CDC. Preliminary estimates of the prevalence of selected underlying health conditions among patents with coronavirus disease 2019- United States, February 12-March 28, 2020. Morb Mortal Wkly Rep. 2020;69:382-6.

9. Clerkin KJ, Fried JA, Raikhelkar J, Sayer G, Griffin JM, Masoumi A, et al. Coronavirus disease 2019 (COVID-19) and cardiovascular disease. Circulation. 2019;2020:2019. https://doi.org/10.1161/ CIRCULATIONAHA.120.046941.

10. Li B, Yang J, Zhao F, Zhi L, Wang X, Liu L, et al. Prevalence and impact of cardiovascular metabolic diseases on COVID19 in China. Clin Res Cardiol. 2020;109:531-538. https://doi. org/10.1007/s00392-020-01626-9.

11. Hu S, Gao R, Liu L, Zhu M, Wang W. Summary of the report on cardiovascular diseases in China. Chin Circ J. 2020;34:209.

12. Zhang Y, Cui Y, Shen M, Zhang J, Liu B, Dai M, et al. Comorbid diabetes mellitus was associated with poorer prognosis in patients with COVID-19: a retrospective cohort study. MedRxiv. 2020. https://doi.org/10.1101/2020.03.24.20042358.

13. Kulcsar KA, Coleman CM, Beck SE, Frieman MB. Comorbid diabetes results in immune dysregulation and enhanced disease severity following MERS-CoV infection. JCI Insight. 2019;4:1-18.

14. Chan JFW, Yuan S, Kok KH, To KKW, Chu H, Yang J, et al. A familial cluster of pneumonia associated with the 2019 novel coronavirus indicating person-to-person transmission: a study of a family cluster. Lancet. 2020;395:514-23. https://doi.org/10.1016/ S0140-6736(20)30154-9.

15. Odegaard JI, Chawla A. Connecting Type 1 and Type 2 diabetes through innate immunity. Cold Spring Harb Perspect Med. 2012;2:a007724.

16. Guan W, Ni Z, Hu Y. China medical treatment expert group for COVID-19. Clinical characteristics of coronavirus disease 2019 in China. N Engl J Med. 2020;382:1708-1720.

17. Stairmand J, Signal L, Sarfati D, Jackson C, Batten L, Holdaway $\mathrm{M}$, et al. Consideration of comorbidity in treatment decision making in multidisciplinary cancer team meetings: a systematic review. Ann Oncol. 2015;26:1325-32.

18. Sarfati D, Gurney J, Lim BT, Bagheri N, Simpson A, Koea J, et al. Identifying important comorbidity among cancer populations using administrative data: prevalence and impact on survival. Asia Pac J Clin Oncol. 2016;12:e47-56.

19. Carstensen J, Andersson D, Andre M, Engstrom S, Magnusson $\mathrm{H}$, Borgquist LA. How does comorbidity influence healthcare costs? A population-based cross-sectional study of depression, back pain and osteoarthritis. BMJ Open. 2012;2:e000809.

20. Sogaard M, Thomsen RW, Bossen KS, Sørensen HT, Nørgaard M. The impact of comorbidity on cancer survival: a review. Clin Epidemiol. 2013;5(Suppl 1):3.

21. Rodriguez-Morales AJ, Cardona-Ospina JA, Gutiérrez-Ocampo E, Villamizar-Peña R, Holguin-Rivera Y, Escalera-Antezana JP, et al. Clinical, laboratory and imaging features of COVID-19: a systematic review and meta-analysis. Travel Med Infect Dis. 2020;34:1-13.

22. Yang J, Zheng Y, Gou X, Pu K, Chen Z, Guo Q, et al. Prevalence of comorbidities and its effects in coronavirus disease 2019 patients: a systematic review and meta-analysis. Int J Infect Dis. 2020;94:91-5.

23. Cheung KS, Hung IFN, Chan PPY, Lung KC, Tso E, Liu R, et al. Gastrointestinal manifestations of SARS-CoV-2 infection and virus load in fecal samples from a Hong Kong Cohort: systematic review and meta-analysis. Gastroenterology. 2020;159:81-95.
24. Alotaibi A, Perry L, Gholizadeh L, Al-Ganmi A. Incidence and prevalence rates of diabetes mellitus in Saudi Arabia: an overview. J Epidemiol Glob Health. 2017;7:211-8.

25. De Toledo IP, Stefani FM, Porporatti AL, Mezzomo LA, Peres MA, Flores-Mir C, et al. Prevalence of otologic signs and symptoms in adult patients with temporomandibular disorders: a systematic review and meta-analysis. Clin Oral Investig. 2017;21:597-605.

26. Munn Z, Moola S, Lisy K, Riitano D, Tufanaru C. Methodological guidance for systematic reviews of observational epidemiological studies reporting prevalence and cumulative incidence data. Int J Evid Based Healthc. 2015;13:147-53.

27. Joanna Briggs Institute (JBI). JBI's critical appraisal tools. Faculty of Health and Medical Sciences, The University of Adelaide SA 5006 Adelaide, Australia. https://joannabriggs.org/ebp/criti cal_appraisal_tools.

28. Higgins JPT, Thompson SG. Quantifying heterogeneity in a metaanalysis. Stat Med. 2002;21:1539-58.

29. Begg CB, Mazumdar M. Operating characteristics of a rank correlation test for publication bias. Biometrics. 1994;50:1088-101.

30. Egger M, Smith GD, Schneider M, Minder C. Bias in meta-analysis detected by a simple, graphical test. BMJ. 1997;31:629-634.

31. Sharp S. sbe23: meta-analysis regression. Stata Tech Bull. 1998;42:16-22.

32. Knapp G, Hartung J. Improved tests for a random effects metaregression with a single covariate. Stat Med. 2003;22:2693-710.

33. Higgins JPT, Thompson SG. Controlling the risk of spurious findings from meta-regression. Stat Med. 2004;23:1663-82.

34. Zhou F, Yu T, Du R, Fan G, Liu Y, Liu Z, et al. Clinical course and risk factors for mortality of adult inpatients with COVID19 in Wuhan, China: a retrospective cohort study. Lancet. 2020;395:1054-62.

35. Wu C, Hu X, Song J, Du C, Xu J, Yang D, et al. Heart injury signs are associated with higher and earlier mortality in coronavirus disease 2019 (COVID-19). MedRxiv. 2020. https://www. medrxiv.org/content/10.1101/2020.02.26.20028589v1

36. Cheng Y, Luo R, Wang K, Zhang M, Wang Z, Dong L, et al. Kidney disease is associated with in-hospital death of patients with COVID-19. Kidney Int. 2020. https://doi.org/10.1016/j. kint.2020.03.005.

37. Fu L, Fei J, Xiang H-X, Xiang Y, Tan Z-X, Li M-D, et al. Influence factors of death risk among COVID-19 patients in Wuhan, China: a hospital-based case-cohort study. medRxiv. 2020. https ://doi.org/10.1101/2020.03.13.20035329.

38. Shi S, Qin M, Shen B, Cai Y, Liu T, Yang F, et al. Association of cardiac injury with mortality in hospitalized patients with COVID-19 in Wuhan, China. JAMA Cardiol. 2020;5:802-810.

39. Guan W, Ni Z, Hu Y, Liang W, Ou C, He J, et al. Clinical characteristics of coronavirus disease 2019 in China. N Engl J Med. 2020;382:1708-1720. https://doi.org/10.1056/NEJMoa2002032

40. Yan Y, Yang Y, Wang F, Ren H, Zhang S, Shi X, et al. Clinical characteristics and outcomes of patients with severe covid-19 with diabetes. BMJ Open Diabetes Res Care. 2020;8:1-9.

41. Wang Z, Yang B, Li Q, Wen L, Zhang R. Clinical features of 69 cases with coronavirus disease 2019 in Wuhan, China. Clin Infect Dis. 2020;71:769-777. https://doi.org/10.1093/cid/ciaa2 72.

42. Pan L, Mu M, Yang P, Sun Y, Wang R, Yan J, et al. Clinical characteristics of COVID-19 patients with digestive symptoms in Hubei, China: a descriptive, cross-sectional, multicenter study. Am J Gastroenterol. 2020;14:115. https://doi. org/10.14309/ajg.0000000000000620.

43. Cao M, Zhang D, Wang Y, Lu Y, Zhu X, Li Y, et al. Clinical features of patients infected with the 2019 Novel coronavirus (COVID-19) in Shanghai, China. medRxiv. 2020. https://doi. org/10.1101/2020.03.04.20030395. 
44. Guan W, Ph D, Liang W, Zhao Y, Med M, Liang H, et al. Comorbidity and its impact on 1,590 patients with COVID-19 in China: A Nationwide Analysis. Eur Respir J. 2020; 55:2000547. https:// doi.org/10.1183/13993003.00547-2020.

45. Chen T, Wu D, Chen H, Yan W, Yang D, Chen G, et al. Clinical characteristics of 113 deceased patients with coronavirus disease 2019: retrospective study. BMJ. 2020. https://doi.org/10.1136/ bmj.m1091.

46. Liu K, Fang Y-Y, Deng Y, Liu W, Wang M-F, Ma J-P, et al. Clinical characteristics of novel coronavirus cases in tertiary hospitals in Hubei Province. Chin Med J (Engl). 2020;133:1025-1031.

47. Wu Z, McGoogan JM. Characteristics of and important lessons from the coronavirus disease 2019 (COVID-19) outbreak in China: summary of a Report of 72314 cases from the Chinese Center for Disease Control and Prevention. JAMA. 2020;2019:3-6.

48. Guo W, Li M, Dong Y, Zhou H, Zhang Z, Tian C, et al. Diabetes is a risk factor for the progression and prognosis of COVID-19. Diabetes Metab Res Rev. 2020:e3319.

49. Wan S, Xiang Y, Fang W, Zheng Y, Li B, Hu Y, et al. Clinical features and treatment of COVID-19 patients in northeast Chongqing. J Med Virol. 2020;92:797-806.

50. Su VYF, Yang Y-H, Yang K-Y, Chou K-T, Su W-J, Chen Y-M, et al. The risk of death in 2019 novel coronavirus disease (COVID-19) in Hubei Province. SSRN eLibrary. 2020:3539655.

51. Lo IL, Lio CF, Cheong HH, Lei CI, Cheong TH, Zhong X, et al. Evaluation of SARS-CoV-2 RNA shedding in clinical specimens and clinical characteristics of 10 patients with COVID-19 in Macau. Int J Biol Sci. 2020;16:1698-707.

52. Memish ZA, Cotten M, Watson SJ, Kellam P, Zumla A, Alhakeem $\mathrm{RF}$, et al. Community case clusters of middle east respiratory syndrome coronavirus in Hafr Al-Batin, Kingdom of Saudi Arabia: a descriptive genomic study. Int J Infect Dis. 2014;23:63-8. https:// doi.org/10.1016/j.ijid.2014.03.1372.

53. Noorwali ASA, Turkistani AHM, Asiri SI, Trabulsi FA, Alwafi OM, Alzahrani SH, et al. Descriptive epidemiology and characteristics of confirmed cases of Middle East respiratory syndrome coronavirus infection in the Makkah Region of Saudi Arabia, March to June 2014. Ann Saudi Med. 2015;35:203-9.

54. Onder G, Rezza G, Brusaferro S. Case-fatality rate and characteristics of patients dying in relation to COVID-19 in Italy. JAMA. 2020;323:1775-1776. https://doi.org/10.1001/jama.2020.4683.

55. Yang X, Yu Y, Xu J, Shu H, Xia J, Liu H, et al. Clinical course and outcomes of critically ill patients with SARS-CoV-2 pneumonia in Wuhan, China: a single-centered, retrospective, observational study. Lancet Respir Med. 2020;2600:1-7. https://doi. org/10.1016/S2213-2600(20)30079-5.

56. Lippi G, Lavie CJ, Sanchis-Gomar F. Cardiac troponin I in patients with coronavirus disease 2019 (COVID-19): evidence from a meta-analysis. Prog Cardiovasc Dis. 2020. https://doi. org/10.1016/j.pcad.2020.03.001.
57. Alqahtani FY, Aleanizy FS, Ali El Hadi Mohamed R, Alanazi MS, Mohamed N, Alrasheed MM, et al. Prevalence of comorbidities in cases of Middle East respiratory syndrome coronavirus: a retrospective study. Epidemiol Infect. 2019;147:4.

58. Mallikethi-Reddy S, Jagadeesh K. Mechanisms of acute coronary syndromes. N Engl J Med. 2013;369:882-3.

59. Wang T, Du Z, Zhu F, Cao Z, An Y, Gao Y, et al. Comorbidities and multi-organ injuries in the treatment of COVID-19. Lancet. 2020;395:e52. https://doi.org/10.1016/S0140-6736(20)30558-4.

60. Gao R, Cao B, Hu Y, Feng Z, Wang D, Hu W, et al. Human infection with a novel avian-origin influenza A (H7N9) virus. N Engl J Med. 2013;368:1888-97.

61. Guo L, Wei D, Zhang X, Wu Y, Li Q, Zhou M, et al. Clinical features predicting mortality risk in patients with viral pneumonia: the MuLBSTA score. Front Microbiol. 2019;11:1304. https://doi. org/10.3389/fmicb.2019.02752.

62. Tan W, Aboulhosn J. The cardiovascular burden of coronavirus disease 2019 (COVID-19) with a focus on congenital heart disease. Int J Cardiol. 2020. https://doi.org/10.1016/j.ijcar d.2020.03.063.

63. Assiri A, Al-Tawfiq JA, Al-Rabeeah AA, Al-Rabiah FA, Al-Hajjar S, Al-Barrak A, et al. Epidemiological, demographic, and clinical characteristics of 47 cases of Middle East respiratory syndrome coronavirus disease from Saudi Arabia: a descriptive study. Lancet Infect Dis. 2013;13:752-61.

64. Yao Y, Tian Y, Zhou J, Ma X, Yang M, Wang S. Epidemiological characteristics of 2019-ncoV infections in Shaanxi, China by February 8, 2020. Eur Respir J. 2020. https://doi.org/10.1183/13993 003.00310-2020.

65. Badawi A, Ryoo SG. Prevalence of comorbidities in the Middle East respiratory syndrome coronavirus (MERS-CoV): a systematic review and meta-analysis. Int J Infect Dis. 2016;49:129-33. https://doi.org/10.1016/j.ijid.2016.06.015.

66. Yang YM, Hsu CY, Lai CC, Yen MF, Wikramaratna PS, Chen $\mathrm{HH}$, et al. Impact of comorbidity on fatality rate of patients with middle east respiratory syndrome. Sci Rep. 2017;7:1-9. https:// doi.org/10.1038/s41598-017-10402-1.

67. Assiri A, McGeer A, Perl TM, Price CS, Al Rabeeah AA, Cummings DAT, et al. Hospital outbreak of middle east respiratory syndrome coronavirus. N Engl J Med. 2013;369:407-16.

68. Assiri A, Abedi GR, Saeed AAB, Abdalla MA, Choudhry AJ, $\mathrm{Lu}$ X, et al. Multifacility outbreak of middle east respiratory syndrome in Taif, Saudi Arabia. Emerg Infect Dis. 2016;22:32-40. http://wwwnc.cdc.gov/eid/article/22/1/15-1370_article. 